Cahiers
Recherche
surles Droits

Fondamentaux
Cahiers de la recherche sur les droits fondamentaux

$8 \mid 2010$

La liberté d'expression

\title{
La protection de la dignité humaine dans le pluralisme juridique contemporain
}

Jean-Guy Belley

\section{OpenEdition}

Édition électronique

URL : https://journals.openedition.org/crdf/6177

DOI : $10.4000 /$ crdf. 6177

ISSN : 2264-1246

Éditeur

Presses universitaires de Caen

Édition imprimée

Date de publication : 20 décembre 2010

Pagination : 117-134

ISBN : 978-2-84133-367-7

ISSN : $1634-8842$

Référence électronique

Jean-Guy Belley, "La protection de la dignité humaine dans le pluralisme juridique contemporain », Cahiers de la recherche sur les droits fondamentaux [En ligne], 8 | 2010, mis en ligne le 08 octobre 2020, consulté le 15 novembre 2022. URL : http://journals.openedition.org/crdf/6177 ; DOI : https://doi.org/ $10.4000 /$ crdf.6177 


\title{
La protection de la dignité humaine dans le pluralisme juridique contemporain
}

\author{
Jean-Guy BELLEY \\ Professeur titulaire de la Chaire William C. Macdonald, Faculté de droit, Université Mc Gill Montréal
}

\begin{abstract}
I. Définitions opératoires et statuts épistémologiques
A. L'idée de pluralisme juridique

B. L'idée de dignité humaine

II. Hypothèse d'évolution

III. La première modernité ou l'épistémè politique de la démocratie libérale

IV. La seconde modernité ou l'épistémè économique du libéralisme corporatif

V. Pour une troisième modernité pluraliste et humaniste
\end{abstract}

Les idées de pluralisme juridique et de dignité humaine connaissent l'une et l'autre aujourd'hui une grande faveur théorique et pratique. Dans un espace mondialisé où d'innombrables processus économiques et sociaux traversent quotidiennement les frontières nationales, il semble aller de soi que la régulation politique et juridique ne saurait être que plurielle ${ }^{1}$. Dans une culture planétaire dominée par la croissance infinie de la richesse matérielle et par l'innovation technologique, il semble évident que le respect des êtres humains ne saurait être assuré que par l'affirmation du droit à la protection de la dignité humaine comme valeur éminente des ordres juridiques nationaux et supranationaux ${ }^{2}$.
La question à laquelle ce texte voudrait répondre est de savoir si les idées contemporaines de pluralisme juridique et de dignité humaine ont entre elles une affinité élective qui les fait se conforter mutuellement ou, au contraire, si elles participent respectivement de manières antinomiques voire antagonistes de concevoir le droit et d'organiser son fonctionnement. En ce début de $\mathrm{XXI}^{\mathrm{e}}$ siècle, dans un contexte de globalisation économique et de technicisme, les promotions simultanées de la pluralité juridique et du respect de la dignité humaine relèvent-elles d'un projet commun et cohérent? Expriment-elles, au contraire, la confrontation dialectique de deux visions opposées de l'avenir souhaitable?

1. C. Parker, «The Pluralization of Regulation», Theoretical Inquiries in Law, 9, 2008, p. 349. Ce texte est une introduction au numéro spécialement consacré au thème suivant: Legal Pluralism, Privatization of Law and Multiculturalism; P. Schiff Berman, « Global legal pluralism », Southern California Law Review, vol. 80, 2007, p. 1155. H. Petersen et H. Zahle (éd.), Legal Polycentricity. Consequences of Pluralism in Law, Dartmouth, Aldershot, 1995.

2. M. Fabre-Magnan, «La dignité en droit: un axiome», Revue interdisciplinaire d'études juridiques, 58, 2007, p. 1. D. Réaume, «Indignities: making a place for dignity in modern legal thought », Queen's Law Journal, vol. 28, n 61, 2002, p. 63. 
Notre réponse procédera de la façon suivante. Nous préciserons d'abord le sens qui sera donné à ces deux idées en montrant qu'elles peuvent être reçues plus ou moins favorablement dans la pensée juridique dominante (I). Nous énoncerons ensuite notre hypothèse selon laquelle la promotion actuelle de ces deux idées dans la théorie générale du droit relève d'une dynamique de conflit impulsée par l'avènement historique d'un pluralisme juridique qui met en péril le respect de la dignité humaine bien davantage qu'il ne le favorise (II). Nous approfondirons cette hypothèse en montrant que la culture politique de la première modernité a permis le renforcement mutuel des idées de monisme juridique et de protection de la dignité humaine (III) tandis que le passage à la seconde modernité favorise nettement l'idée de pluralisme juridique, mais pourrait être néfaste à celle de dignité humaine (IV). Nous terminerons en nous interrogeant sur les changements qui pourraient être apportés à l'ordre actuel des choses pour que les efforts respectifs en faveur du pluralisme juridique et du respect de la dignité humaine convergent au lieu de s'opposer (V).

\section{Définitions opératoires et statuts épistémologiques}

\section{A. L'idée de pluralisme juridique}

L'idée de pluralisme juridique sera entendue dans ce texte comme élément caractéristique d'une conception générale en vertu de laquelle est postulée ou reconnue l'existence simultanée d'une pluralité d'ordres normatifs ayant le statut de «droit» dans un espace-temps donné. Dès lors que ce pluralisme juridique est admis se pose inévitablement la question de savoir quels rapports de principe (depuis l'indépendance jusqu'à la subordination en passant par divers degrés d'autonomie et d'intégration $)^{3}$ et quelles relations de fait (depuis la coexistence passive jusqu'à la collaboration active en passant par divers états de complémentarité, de concurrence et de conflit) ${ }^{4}$ devraient avoir et ont entre eux les divers ordres juridiques reconnus.

À sa face même, notre définition peut couvrir une variété considérable de conceptions différentes voire opposées du pluralisme juridique, selon le type d'espacetemps envisagé (prémoderne/ moderne; politique / économique...), selon la manière d'attribuer le statut de «droit» qui distingue l'ordre juridique de l'ordre simplement normatif (l'attribut juridique qui fait la différence est-il une qualité socialement reconnue dans l'espace- temps considéré ou plutôt le construit analytique d'un observateur externe?), selon que la théorie proposée se veut normative (il s'agit de faire admettre telle ou telle conception dogmatique des rapports de principe entre les ordres juridiques), critique (la théorie avancée veut contribuer à promouvoir un changement dans la distribution des statuts et/ou des forces entre les ordres en présence) ou empirique / heuristique (la pluralité des ordres juridiques est posée comme un fait empiriquement observable ou postulée comme une voie d'analyse plus adéquate que la représentation dogmatique en vigueur ou que celle entretenue par les acteurs œuvrant dans l'espace-temps considéré pour expliquer/comprendre la dynamique concrète de la régulation juridique).

Il n'entre pas dans le propos de ce texte de recenser les diverses théories du pluralisme juridique formulées à ce jour, dans la science du droit et dans les sciences sociales, ou d'en proposer une nouvelle conception ${ }^{5}$. Nous nous limiterons plutôt à la mise en évidence et à l'illustration sommaire des trois statuts épistémologiques différents qui peuvent être conférés à l'idée de pluralisme juridique. Nous utiliserons cette distinction dans la section suivante pour caractériser le changement qui s'opère aujourd'hui du point de vue de son importance sociale.

L'idée de pluralisme juridique jouit du statut épistémologique - ou mieux peut-être épistémique - le plus important socialement lorsqu'elle occupe une position centrale dans la culture commune des acteurs de l'espacetemps considéré et surtout dans la doctrine officielle soutenue par les dirigeants et les professionnels auxquels est reconnu le pouvoir légitime d'énoncer ou d'appliquer le «droit». On peut considérer, par exemple, que, dans l'Europe du Moyen Âge, les ordres juridiques des organisations ecclésiastiques, des cités, des corporations de métiers, des classes sociales et autres groupes d'appartenance ou de référence jouissaient en principe d'un statut indépendant ou autonome vis-à-vis de l'ordre juridique de l'État, en vertu d'une culture politique et d'une doctrine juridique gagnées à l'idée de pluralisme juridique ${ }^{6}$.

Moins assuré socialement puisqu'il participe de luttes de pouvoir et fait l'objet de débats doctrinaux se trouve le statut d'idéologie sociale qu'acquiert l'idée de pluralisme juridique lorsqu'elle est mobilisée comme justification normative et caution théorique de la résistance d'un groupe contre une diminution de ses pouvoirs ou de la revendication pour une augmentation de ses attributions. L'invocation de la doctrine médiévale du pluralisme juridique par l'Église catholique luttant contre la perte de ses prérogatives juridiques au profit de l'État moderne ${ }^{7}$ et la

3. G. Gurvitch, L'Idée du droit social, Paris, Sirey, 1932, réimpr. Verlag, 1972, p. 53-95 (typologie des formes de droit social envisagées dans leurs rapports de principe avec l'ordre juridique de l'État).

4. J. Griffiths, "What is legal pluralism?», Journal of Legal Pluralism, vol. 24, 1986, p. 1-55 (typologie des relations de fait entre des ordres juridiques participant d'un ensemble qui ne gravite pas nécessairement autour d'un ordre juridique central).

5. Voir notamment K. Walby, «Contributions to a post-sovereigntist understanding of law : Foucault, law as governance, and legal pluralism », Social and Legal Studies, 2007, p. 551; aussi J.-G. Belley, «L'État et la régulation juridique des sociétés globales. Pour une problématique du pluralisme juridique », Sociologie et Sociétés, XVIII, 1986, p. 11-32.

6. A.-J. Arnaud, Pour une pensée juridique européenne, Paris, PUF, 1991, p. 55-100.

7. A. Girard, J. Tonneau et L. Lachance, «La Justice», in Initiation théologique par un groupe de théologiens, t. III, Théologie morale, Paris, Les Éditions du Cerf, 1952, p. 723-858 (779) : «Ici encore, il convient d'assouplir notre conception moderne de la vie sociale, en renonçant au fétichisme de l’État et de la 
mobilisation d'une théorie ethnologique du pluralisme juridique (persistance des droits locaux, coutumiers, ancestraux, inofficiels) au soutien des luttes pour la décolonisation contre les métropoles européennes au cours des deux ou trois derniers siècles ${ }^{8}$ sont des illustrations parmi d'autres d'une idée de pluralisme juridique faisant office d'idéologie sociale.

Le troisième statut épistémologique est celui de construit scientifique. Au sein d'une discipline savante, dans la théorisation généralement admise ou dans un courant minoritaire contestant le bien-fondé des a priori de la science normale, l'idée de pluralisme juridique est élevée au rang de postulat ou exprimée sous forme de concept scientifique. Ce statut ne confere pas au pluralisme juridique l'autorité doctrinale d'une prétention valide en droit et tenue pour vraie dans la pensée juridique dominante. Il ne lui procure même pas la visibilité voire la légitimité d'une idéologie sociale exprimée publiquement par une collectivité plus ou moins puissante. En revanche, lorsqu'elle acquiert le statut d'un construit scientifique, l'idée de pluralisme juridique a déjà bénéficié d'une systématisation souvent plus poussée que ce n'est le cas pour une doctrine juridique et, a fortiori, pour une idéologie sociale. Elle a aussi l'avantage de pouvoir se réclamer de la légitimité particulière d'une vérité scientifique qui s'imposerait au nom de sa conformité à la réalité objective des choses plutôt qu'en vertu d'une vision idéale soutenue par la métaphysique juridique officielle ou par la force arbitraire d'un acteur du jeu politique.

La faveur qu'a connue l'idée de pluralisme juridique dans le champ des études «droit et société » depuis 1960 est une illustration très manifeste de son ascension au statut de construit scientifique. Bénéficiant au départ d'une adhésion minoritaire mais se réclamant du prestige des plus grands précurseurs de la sociologie et de l'anthropologie du droit ${ }^{9}$, l'idée est aujourd'hui solidement implantée en théorie et dans la recherche empirique. Qui plus est, son rayonnement s'étend désormais à l'intérieur même de la science juridique, chez des juristes de plus en plus nombreux à y voir non plus une hérésie venant des sciences sociales, mais plutôt une représentation adéquate de la complexité du droit positif contemporain et la valorisation souhaitable d'une organisation pluraliste plutôt que moniste de la régulation juridique à mettre en place pour assumer les défis du XXI ${ }^{e}$ siècle. Il est peut-être prématuré de prétendre que le pluralisme juridique fait désormais office de doctrine officielle dans la théorie générale du droit. Mais, il est certainement plausible qu'elle acquière ce statut dans un avenir assez rapproché, tant il est vrai que l'idée fait déjà consensus dans les branches spéciales de la science du droit dont le développement et l'influence croissante sont favorisés par l'évolution actuelle de la société, de l'économie et de la politique $^{10}$.

Quoi qu'il en soit, la migration du postulat ou du concept de pluralisme juridique du champ «droit et société» vers le champ juridique ${ }^{11}$ montre que les trois statuts que nous venons de distinguer ne s'excluent pas mutuellement et peuvent même combiner leurs effets pour maximiser l'importance sociale de l'idée. On imagine facilement, par exemple, que, mobilisée idéologiquement par un groupe social opprimé ou conquérant, l'idée trouve des échos empathiques ou accommodants dans telle ou telle communauté savante et que sa promotion scientifique finisse par influencer la pensée juridique en lui facilitant le travail de construction doctrinale. Tel nous semble être le cas dans la seconde modernité contemporaine comme nous le suggérerons plus loin. Nous verrons auparavant que l'idée de dignité humaine peut, elle aussi, connaître successivement ou concurremment ces trois statuts épistémologiques.

\section{B. L'idée de dignité humaine}

Nous considérerons ici que, pour l'essentiel, l'idée de dignité humaine affirme la valeur éminente de tout être humain reconnu comme une personne à part entière, indépendamment de ses caractéristiques individuelles et de ses appartenances sociales. Cette idée a clairement acquis le statut d'une doctrine juridique officielle dans les sociétés occidentales modernes marquées par

loi écrite. Il est bon de se remettre dans la perspective médiévale d'un pluralisme juridico-social extrêmement compliqué et divers, avec une multitude de communautés jouissant d'un droit particulariste, selon les coutumes locales, les statuts personnels et les engagements contractés. » J.-G. Belley, «Une croisade intégriste chez les avocats du Québec: La Revue du droit (1922-1939) », Les Cahiers de Droit, 34, 183, 1993, p. 195 sq. (« Un pluralisme juridique du type théocratique»).

8. É. Le Roy, «Sur les chemins de Kahnawake. Décolonisation du droit et mondialisations », in Mélanges Andrée Lajoie. Le droit, une variable dépendante, P. Noreau et L. Rolland (dir.), Montréal, Thémis, 2008, p. 493-524.

9. P. Selznick, «The sociology of law », in International Encyclopedia of the Social Sciences, vol. 9, New York, Macmillan - The Free Press, 1968, p. 50-59 (50-51). J.-G. Belley, Conflit social et pluralisme juridique en sociologie du droit, thèse de doctorat de $3^{\mathrm{e}}$ cycle, Université de droit, d'économie et de sciences sociales de Paris, 1977, p. 43-83.

10. J.-G. Belley, «Le pluralisme juridique comme doctrine de la science du droit », in Pour un droit pluriel. Études offertes au professeur Jean-François Perrin, J. Kellerhals et al. (dir.), Genève, Helbing \& Lichtenhahn, 2002, p. 135-165. La réhabilitation du pluralisme juridique comme doctrine de la science du droit n'est évidemment pas sans rapport avec la critique néolibérale du droit étatique et des institutions de la démocratie formelle, critique dont les organisations et réseaux de la mondialisation économique sont les principaux bénéficiaires. Voir, en ce sens, L. Cohen-Tanugi, Le droit sans l'État: sur la démocratie en France et en Amérique, Paris, PUF, 1985. Pour une critique de cette version très normative du pluralisme juridique, voir G. Barzilai, «Beyond relativism: where is political power in legal pluralism», Theoretical Inquiries in Law, 9, 2008, p. 395. J. Weber, «Legal pluralism and human agency ", Osgoode Hall Law Journal, 167, 44, 2006, p. 180-182. La réhabilitation néolibérale du pluralisme juridique va aussi de pair avec la reféodalisation des rapports juridiques établis à travers des contrats de dépendance dont les grands groupes privés sont les maîtres d'œuvre et premiers bénéficiaires. Voir, à ce sujet, A. Supiot, Homo Juridicus. Essai sur la fonction anthropologique du droit, Paris, Seuil, 2005, p. 164-165.

11. J.-G. Belley, «Le droit comme terra incognita : conquérir et construire le pluralisme juridique », Revue canadienne / droit et société, vol. 12 , $\mathrm{n}^{\circ} 2$, 1997, p. 11-14. 
l'avènement révolutionnaire ou non d'un État se réclamant d'une constitution démocratique ${ }^{12}$.

En postulant la transcendance de l'État comme représentant d'un intérêt général supérieur à tous les intérêts privés, le constitutionnalisme démocratique rend possible du même coup l'affirmation d'une égale dignité de tous les êtres humains fondée sur leur appartenance commune à une même collectivité abstraite, la Nation. Au-delà de toutes les formes d'inégalité (sociale, économique, culturelle...) qui les distinguent concrètement, le partage d'une nationalité ou citoyenneté commune confère à tous les détenteurs de ce statut une même égalité juridique de principe. La doctrine de l'égale dignité de tous les citoyens d'un même État démocratique est la version juridique moderne de l'ancienne doctrine chrétienne de l'égale valeur de tous les "enfants de Dieu», indépendamment de leur statut social ou de leur richesse.

Aussi bien après qu'avant l'émergence de l'État démocratique, l'idée de dignité humaine se retrouve au cœur d'un grand nombre d'idéologies sociales. Elle fut mobilisée au nom de l'accès de tous à la pleine reconnaissance de leur statut de citoyens. Songeons ici aux mouvements prônant l'égalité juridique des femmes, des enfants et des membres des minorités de toutes sortes. Elle a aussi été invoquée pour justifier une extension de l'idée de citoyenneté et de ses effets juridiques, soit au-delà de la liste classique des droits fondamentaux (les revendications en faveur de la constitutionnalisation des droits économiques et sociaux...), soit au-delà de la sphère des rapports entre l'État et les citoyens, au sein de la société civile et dans les rapports entre sujets de droit. L'exemple le plus connu de cette seconde extension est celui des revendications ouvrières en faveur de la reconnaissance d'une «citoyenneté économique» ou d'une « démocratie industrielle» qui affirmerait la dignité des travailleurs comme limite aux prérogatives des propriétaires des moyens de production et des détenteurs de capitaux ${ }^{13}$.

Dans les idéologies sociales qui viennent d'être évoquées, la revendication de droits nouveaux au nom de la dignité humaine prenait appui directement sur le statut de citoyens d'un État démocratique. La citoyenneté servait de levier pour revendiquer auprès de l'État d'appartenance les lois ou autres interventions qui protégeraient la dignité humaine en améliorant la condition concrète des consommateurs, travailleurs, prestataires d'aide sociale ou usagers des services publics du pays.

Il n'en va pas de même dans les idéologies contemporaines qui invoquent le respect de la dignité humaine en faveur de personnes qui n'ont pas la citoyenneté du pays visé, qui sont citoyennes d'un État non officiellement démocratique ou qui revendiquent un droit auquel même les citoyens à part entière ne peuvent prétendre. Pensons notamment aux mouvements de défense des réfugiés, immigrants, étrangers sans papiers, apatrides, prisonniers de guerre, victimes civiles de conflits armés, condamnés en attente d'exécution d'une peine capitale...

Le point d'appui de ces diverses revendications humanitaires ou philanthropiques réside dans l'invocation de la commune humanité des personnes à protéger plutôt que d'une citoyenneté commune. L'appartenance mobilisée idéologiquement se conçoit comme universelle plutôt que nationale. C'est à titre de membre du genre humain que la personne devrait bénéficier du respect de la dignité humaine. Outrepassant les frontières du constitutionnalisme démocratique, la revendication humanitaire trouve dans la tradition philosophique la conception de la dignité humaine dont pourraient se réclamer tous ceux que même les États les plus démocratiques laissent sans voix pour exprimer leurs souffrances et sans pouvoir pour contrer l'oppression dont ils sont victimes.

Il n'est pas étonnant que la philosophie ait été et reste la principale source d'inspiration des personnes et des mouvements sociaux qui se réclament d'une dignité humaine à prétention universelle. Puisque la prétention à l'égale dignité de tous les êtres humains requiert l'affirmation de leur appartenance commune à une même collectivité abstraite, puisque les anciennes abstractions de la « communauté chrétienne» ou de la «nation» ne suffisent pas à transcender la diversité des croyances religieuses et des appartenances nationales, la construction théorique de l' " humanité » comme collectivité abstraite d'envergure universelle ne pouvait guère s'effectuer en dehors de la philosophie et de ses prétentions à s'élever

12. Depuis l'apparition explicite du mot «dignité» dans le langage formel du droit international et des droits nationaux, soit depuis la fin de la seconde guerre mondiale, les juristes ont appris à distinguer une acception large du concept (l'égale dignité de tous les êtres humains comme valeur générale qui se trouve au fondement de toutes les libertés et de tous les droits constitutionnellement garantis) et une acception étroite (le droit au respect de la dignité opposable à un acte gouvernemental ou à une pratique privée qui dévalorise un individu ou un groupe, porte atteinte à son estime de soi, perpétue des préjugés ou des stéréotypes irrespectueux). La Charte des droits et libertés de la personne du Québec, L.R.Q. c. C-12, par exemple, a retenu ces deux acceptions, respectivement au quatrième alinéa de son Préambule ( Considérant que le respect de la dignité de l’être humain et la reconnaissance des droits et libertés dont il est titulaire constituent le fondement de la justice et de la paix») et en son article 4 ( « Toute personne a droit à la sauvegarde de sa dignité, de son honneur et de sa réputation »). Dans le présent texte, la dignité humaine sera entendue dans son sens large qui en fait une aspiration politico-juridique transcendante, plutôt que dans le sens étroit qui lui confère le statut d'un droit subjectif autonome qui, au même titre que les autres droits et libertés fondamentaux, protège un intérêt patrimonial ou extrapatrimonial spécifique, notamment l'intégrité physique ou la réputation. En tant qu'aspiration démocratique, la dignité relève principalement du droit public. En tant que protection de la personne contre la dévalorisation, la dignité relève tout autant du droit des obligations civiles. Sur le concept de dignité et les dangers afférents aux analyses juridiques qui n'en distinguent pas suffisamment les acceptions large et étroite, voir D. Proulx, «La dignité : élément essentiel de l'égalité ou cheval de Troie? », in Les 25 ans de la Charte canadienne des droits et libertés, Barreau du Québec, Service de la formation continue, Cowansville, Éditions Yvon Blais, 2007, p. 95121; aussi du même auteur, «Le concept de dignité et son usage en contexte de discrimination : deux Chartes, deux modèles », Revue du Barreau du Québec, numéro spécial, 2003, p. 485-542.

13. M. Coutu, «Legal pluralism, labour law and the sovereign state», Canadian Labour \& Employment Law Journal, 13, 2007, p. 169-185. Sur les défis que la mondialisation de l'économie pose au maintien des gains juridiques obtenus depuis le milieu du XX $\mathrm{X}^{\mathrm{e}}$ siècle en faveur de la « démocratie industrielle», voir K. V. W. Stone, «Flexibilization, globalization and privatization : three challenges to labour rights in our time», Osgoode Hall Law Journal, 44, 2006, p. 77-104. 
abstraitement au-dessus des contingences ou des différences concrètes mises en évidence par des sciences sociales moins métaphysiques comme la sociologie et l'anthropologie ${ }^{14}$.

Antérieurement et parallèlement à la consécration juridique des doctrines chrétienne et démocratique, la tradition philosophique s'est employée à postuler l'unité du genre humain qui se distinguerait du monde des dieux, des êtres vivants du monde animal et des entités abstraites portées à l'existence par l'esprit humain mais dépourvues d'un substrat biologique (pensons ici de façon toute particulière à la distinction entre les personnes «morales » et les personnes "physiques»). Depuis l'Antiquité jusqu'à aujourd'hui, de nombreuses variantes de ce que nous appellerons l' «humanisme philosophique» ont prétendu dépasser les limitations des doctrines religieuses et politiques en fondant l'appartenance commune à l'humanité sur l'aptitude universelle des êtres humains à l'exercice de la raison réflexive et de la conscience morale. Cette aptitude leur conférerait potentiellement une manière spécifique de concevoir le monde physique et le rapport à autrui, d'agir et même de souffrir au sein de l'univers.

Bien qu'il ait inspiré des proclamations de principe comme la Déclaration universelle des Droits de l'homme, l'humanisme philosophique n'a pas encore atteint le statut d'une doctrine juridique qui s'imposerait péremptoirement à la doctrine moderne de la souveraineté nationale. La reconnaissance formelle de l'unité politique du monde reste insuffisante pour fonder en droit la prétention d'une instance supranationale à une souveraineté universelle supérieure à celle des États-Nations ${ }^{15}$. Même si cette aspiration gagne du terrain à la faveur d'un mouvement humanitaire d'envergure mondiale, on ne peut pas non plus tenir pour certain le triomphe politique de cette idéologie sociale transnationale sur les mouvements concurrents qui se réclament des identités religieuses ou nationales plus anciennes.

La destinée politique, juridique et sociale de la conception humaniste de la dignité humaine est d'autant plus incertaine aujourd'hui qu'elle se joue dans le contexte épistémique d'une seconde modernité qui bouleverse les catégories philosophiques les plus fondamentales, notamment celles de la raison et de la conscience morale. Dans une culture planétaire dominée par la technique et la présence de machines dont le fonctionnement échappe au sens commun bien que conçu par l'esprit humain, on ne peut plus tenir pour une évidence catégorique que l'être humain reste au centre du monde et que sa prééminence morale soit incontestable. Dépassé par ses propres créations, l'esprit humain perd graduellement sa propension à soutenir une vision anthropomorphique du monde. La pensée technique et la culture nouvelle du rapport nécessaire de l'être humain aux machines, qui ne prolongent plus simplement son action mais qui l'englobent, confrontent l'humanisme philosophique en rendant plausible l'hypothèse d'une «obsolescence de l'humanité » ${ }^{16}$.

\section{Hypothèse d'évolution}

L'hypothèse qui sera approfondie dans ce texte est celle d'une évolution contemporaine marquée par une dynamique de conflit plutôt que de renforcement mutuel entre la promotion du pluralisme juridique et la protection de la dignité humaine. Cette évolution se réaliserait à travers le passage de la première modernité $\left(\mathrm{XVII}^{\mathrm{e}}\right.$ au XIX ${ }^{\mathrm{e}}$ siècle) à la seconde (XX ${ }^{\mathrm{e}}$ et $\mathrm{XXI}^{\mathrm{e}}$ siècles).

Depuis le traité de Westphalie (1648) jusqu'à la fin du XIX ${ }^{e}$ siècle, l'évolution constitutionnelle s'était faite dans le sens de l'affirmation d'un État souverain obéissant de plus en plus formellement aux exigences d'un régime de démocratie libérale. À ce stade de la modernité, l'idée de pluralisme juridique perd beaucoup de l'importance sociopolitique qu'elle avait au Moyen Âge. L'idée d'un monisme juridique organisé hiérarchiquement sous l'égide du droit souverain de l'État remplace le pluralisme juridique comme doctrine ou théorie générale du droit. Le statut de l'idée de pluralisme juridique sera dès lors celui d'une idéologie sociale de résistance à la montée en puissance de l'État et celui d'une critique scientifique formulée à l'encontre de la doctrine moniste officielle au nom du contrôle effectif que beaucoup de groupements anciens ou nouveaux conserveraient sur la régulation quotidienne des activités et des personnes relevant de leurs juridictions.

En revanche, l'idée de dignité humaine voit croître son importance sociale au fur et à mesure que s'affirment l'unité et la centralité de l'État-Nation au détriment de la reconnaissance juridique de la diversité sociale et des anciennes hiérarchies. Le monisme juridique et la protection de la dignité se confortent mutuellement puisque

14. Sur l'évolution de la doctrine des droits fondamentaux vers l'idée contemporaine d'une égale dignité des êtres humains reposant sur leur humanité commune (humanness), voir M. Mautner, «From "Honor" to "Dignity" : how should a liberal State treat non-liberal cultural groups? ", Theoretical Inquiries in Law, 9, 2008, p. 609; sur les effets possiblement négatifs de la référence à une culture universelle de la dignité humaine qui ne prend pas suffisamment en compte les particularités des cultures locales, voir S. E. Merry, « Human Rights and transnational culture : regulating gender violence through global law», Osgoode Hall Law Journal, 44, 2006, p. 53-75.

15. À titre d'exemple, citons le paragraphe suivant de la Convention internationale globale et intégrée pour la protection et la promotion des droits et de la dignité des personnes handicapées, Nations Unies, E/2003/12: "Soulignant que c'est aux gouvernements qu'incombe la responsabilité principale de la promotion et de la protection de tous les droits fondamentaux et libertés fondamentales des personnes handicapées et du plein exercice de ces droits et libertés par les personnes handicapées.»

16. A. Haesler, "Obsolescence de l'homme», in Dictionnaire des risques, Y. Dupont (dir.), Paris, Armand Colin, 2003, p. 282-286. L'auteur expose la thèse du philosophe Günther Anders (1902-1992) à l'effet que le «technomorphisme » contemporain condamne l'être humain à la « honte prométhéenne»: «Être imparfait et non fiable dans un univers d'objets de plus en plus performants, être manquant d'imagination pour voir et prévoir les conséquences de son action, être jeté innocemment dans un monde qu'il doit détruire pour paradoxalement y survivre, le seul sentiment correspondant à cette série de frustrations existentielles est la honte» (p. 285). 
l'aspiration de l'État à la souveraineté appelle en contrepartie son obligation constitutionnelle de respecter la liberté et ultimement la dignité des citoyens partenaires du contrat social fondateur.

Le passage à la seconde modernité a remis fondamentalement en question cet équilibre synergique entre le monisme juridique étatique et l'individualisme libéral. En droit comme en fait, le $\mathrm{XX}^{\mathrm{e}}$ siècle aura vu proliférer comme jamais auparavant les personnes morales et organisations de toutes sortes qui réintroduisent entre l'État et les individus des entités collectives particularistes jalouses de leur autonomie juridique face à l'État et soucieuses de s'assurer la loyauté de leurs agents et de leurs membres ou bénéficiaires. La centralité de l'État et la primauté de la liberté individuelle paraissent encore plus contingentes en ce début de $\mathrm{XXI}^{\mathrm{e}}$ siècle alors que la mondialisation économique semble exiger tout à la fois que l'État renonce à son ancienne souveraineté juridique et que les organisations transnationales ou multinationales se voient reconnaître un statut plus affirmé dans la régulation effective de processus transfrontières qui échappent au pouvoir d'initiative et de contrôle des personnes physiques.

Dans ce contexte, la table est mise pour le remplacement du régime de la démocratie libérale par un régime de libéralisme corporatif ${ }^{17}$ qui change radicalement le statut respectif des idées de pluralisme juridique et de dignité humaine. Dans une société et une économie d'organisations ${ }^{18}$, comme d'ailleurs au sein de l'État devenu luimême un conglomérat complexe d'entités administratives spécialisées et autonomes, l'accession de l'idée de pluralisme juridique au rang de doctrine officielle paraît incontournable pour traduire adéquatement la répartition effective des pouvoirs et des responsabilités et surtout pour augmenter les bénéfices de la spécialisation fonctionnelle et de la mondialisation de l'action économique, sociale et politique.

Pour que ces bénéfices adviennent, il convient en contrepartie que la liberté acquise par les organisations ou les entités corporatives vis-à-vis de l'État-Nation ne soit pas entravée par le pouvoir de blocage juridique concédé en principe aux individus dans l'ancienne démocratie libérale. Le statut juridique de l'idée de dignité humaine ne peut qu'en être affecté à la baisse. Le discours juridique officiel, notamment celui des instances judiciaires supérieures invoquant haut et fort les principes des chartes des droits fondamentaux, confère encore à la dignité humaine une grande charge symbolique. Mais, les règles du droit positif en vigueur et les interventions quotidiennes des autorités publiques confirment clairement une politique juridique générale favorable à l'aménagement de pouvoirs organisationnels ou corporatifs que la protection des libertés individuelles a vocation à infléchir, mais sans jamais entraver leur efficacité.
Cette politique juridique de la seconde modernité n'est qu'une manifestation particulière de la culture contemporaine qui promeut généralement une vision technique du monde et de l'action. Quand tout s'envisage comme une ressource potentiellement utile à la transformation de l'ordre des choses, non seulement la matière, mais aussi les êtres vivants et les productions de l'esprit humain, rien ne s'oppose plus à ce que les inventions de la pensée juridique, notamment la catégorie de «personne morale », soient aussi considérées comme des techniques mises au service de l'action efficace et affranchies des anciennes limitations de l'humanisme individualiste. Le respect de la dignité humaine doit dès lors s'entendre dans un sens compatible avec la promotion du technicisme juridique à usage organisationnel ou collectiviste.

Les deux sections suivantes approfondiront cette hypothèse d'évolution générale au terme de laquelle le pluralisme juridique deviendrait la nouvelle orthodoxie juridique tandis que le respect de la dignité humaine ne serait plus qu'un principe juridique correcteur plutôt que directeur et peut-être même une idéologie sociale de résistance au technicisme davantage qu'une valeur éminente du droit.

\section{La première modernité ou l'épistémè politique de la démocratie libérale}

À ce stade dont la fin du XIX ${ }^{\mathrm{e}}$ siècle marque l'apogée doctrinal, la culture juridique officielle est dominée par la représentation d'un face-à-face entre l'État détenteur de la souveraineté juridique et l'individu considéré comme citoyen et sujet de droit.

Cette vision juridique du monde est le produit de deux grandes évolutions historiques. La première est celle de la décolonisation, c'est-à-dire de l'accession d'un droit national à l'indépendance juridique au détriment d'un ancien ordre juridique impérial ou métropolitain. L'affirmation du nationalisme juridique produit des effets différents selon le niveau de régulation envisagé. À l'échelle mondiale, le pluralisme juridique s'accroît par l'augmentation du nombre des États souverains. À l'échelle nationale, qui est désormais celle que privilégie la science du droit, la décolonisation conforte logiquement la doctrine moniste en vertu de laquelle l'État souverain décide, à l'extérieur, de son degré de participation à la communauté internationale, et, à l'intérieur, du degré d'indépendance ou d'autonomie de tout ordre normatif, y compris religieux, œuvrant sur le territoire national. À l'échelle individuelle, la décolonisation juridique désaliène le moi anciennement colonisé. Elle rend possible la construction d'un moi citoyen plus ou moins confortable selon que l'individu appartient à la majorité nationale ou à une minorité culturelle du pays.

17. Sur l'idéologie du corporate liberalism et le rôle décisif joué par les institutions juridiques dans son triomphe au XX ${ }^{\mathrm{e}}$ siècle, voir S. R. Bowman, The Modern Corporation and American Political Thought. Law, Power and Ideology, University Park, The Pennsylvania State University Press, 1996. Voir aussi R. J. Lustig, Corporate Liberalism: The Origins of Modern American Political Theory, 1890-1920, Berkeley, University of California Press, 1982.

18. R. Presthus, The Organizational Society, New York, Random House, 1962 ; C. Ménard, L'Économie des organisations, Paris, La Découverte, 199o. 
La seconde évolution historique favorable au régime de la démocratie libérale est la délocalisation par laquelle s'affirment la construction et la prééminence juridique, économique et politique d'un seul espace national sur la pluralité des espaces locaux. La doctrine moniste des sources du droit et de la hiérarchie des régulations juridiques décrète la primauté du droit commun du pays (common law of the land) sur les coutumes locales; elle confère à l'ordre juridique de l'État la qualité de droit prépondérant (Higher Law) à l'encontre de tout ordonnancement juridique local ou particulariste. La délocalisation de la culture juridique dominante fait disparaître l'ancien pluralisme juridique infraétatique ou plus exactement le fait apparaître désormais, aux fins du droit, comme un pluralisme juridique concédé ou toléré par l'État, intégré ou annexé par lui et soumis à son pouvoir de surveillance et de contrôle plutôt qu'indépendant ${ }^{19}$.

En revanche, la délocalisation, ou son envers positif la centralisation nationale du droit, favorise l'émancipation juridique des individus. Elle libère ou désaliène le moi traditionnel et communautaire au profit du moi moderne et individualiste. La qualité de sujet de droit au sein de l'ordre juridique étatique confère des droits politiques (les libertés publiques fondamentales), judiciaires (liberté d'ester en justice devant les tribunaux de l'État) et civils (le droit à la propriété privée individuelle, la liberté de contracter et d'entreprendre, la liberté testamentaire...) qui pourront être invoqués à l'encontre de la culture traditionnelle et opposés à l'autorité des groupes d'appartenance. La personnalité juridique des individus est plus affirmée et soutenue dans le droit libéral que celle des groupes et autres collectivités. Le moi idéal de la première modernité est le moi bourgeois: plus national que l'ancien moi aristocratique; plus actif et plus libre économiquement, politiquement et juridiquement que le moi paysan ou le moi ouvrier.

Pénétrons maintenant plus profondément à l'intérieur de la culture juridique de la première modernité pour mieux en identifier les représentations constitutives et comprendre le statut qu'elles confèrent respectivement aux idées de pluralisme juridique et de dignité humaine.

La nouvelle épistémè politique situe l’État au centre de la société. À travers ses organes représentatifs, il se conçoit comme le corps symbolique de la Nation réunie en assemblée démocratique de citoyens égaux. Il est la personne morale par excellence, celle qui détient le pouvoir souverain de faire naître ou disparaître juridiquement la personnalité morale des groupes ou autres entités quelles que soient leurs prétentions à l'importance sociale ou économique.
Dans cette vision des choses, le droit positif de l'État devient le modèle standard de la juridicité. C'est un droit formaliste qui exprime, à travers la loi ou la jurisprudence des tribunaux judiciaires, la volonté de l'État souverain. C'est un droit d'application générale. Il se décline en droit public pour la régulation des rapports avec les citoyens et en droit privé commun à l'ensemble des actes juridiques individuels à travers lesquels se réalise une décentralisation de la régulation juridique pour autant que les formes institutionnelles et les règles substantives prévues par le droit étatique soient respectées. L'ordonnancement du régime est celui d'un système juridique dont le fonctionnement obéit à une autorité pyramidale. La validité des actes publics et privés s'apprécie en fonction d'une légalité souveraine dont les prescriptions et modèles symboliques pénètrent de haut en bas et du centre vers la périphérie tous les niveaux et toutes les manifestations de la vie juridique ${ }^{20}$.

Cette culture juridique se représente l'être humain comme une personne dotée d'un corps physique soumis au contrôle d'une âme capable de rationalité et de civisme. Cette vision abstraite et quasi métaphysique fait de chaque être humain un individu singulier et une entité non décomposable qu'on ne peut priver ni de son corps, protégé par le droit à l'intégrité, ni de son âme, protégée par le droit à la dignité, sans porter une atteinte illicite aux attributs inaliénables (indisponibles) et imprescriptibles de la personne.

La personnalité juridique est la dimension supplémentaire que l'ordre symbolique du droit ajoute spécifiquement à la conception humaniste de l'être humain en se substituant à l'ordre symbolique de la religion. Le statut de «personne juridique» confere l'ensemble des droits constitutionnels et civils qui permettent à l'être humain de satisfaire ses besoins individuels tout en contribuant par son comportement rationnel et civique au règne de la Raison et de la Loi dans la Cité. Cette personnalité juridique advient de façon originaire et non dérivée: chacun en jouit d'emblée du simple fait d'exister comme être humain, indépendamment des groupes auxquels il appartient et des relations qu'il entretient avec autrui. La conception moderne de la personnalité juridique est atomiste et individualiste. Elle se démarque radicalement de la conception communautaire et relationnelle entretenue par les ordres normatifs traditionnels ${ }^{21}$.

Dans le face-à-face symbolique où l'État souverain et l'individu libre se constituent mutuellement, les atteintes à la dignité humaine se ramènent essentiellement à deux types principaux. Elles sont juridiques si elles découlent des actes législatifs, exécutifs ou judiciaires de l'État. Le principe général de la légalité protège l'individu

19. À titre d'exemple de cette représentation d'un pluralisme juridique en principe intégré et surveillé au sein de l'ordre juridique global de l'État, l'article 33 du Code de procédure civile du Québec : « [...] les tribunaux relevant de la compétence du Parlement du Québec, ainsi que les corps politiques, les personnes morales de droit public ou de droit privé au Québec, sont soumis au droit de surveillance et de réforme de la Cour supérieure [sauf exceptions expresses] [...]»

20. Sur les racines religieuses de cette juridicité étatique intrusive, voir T. Murphy, The Oldest Social Science? Configurations of Law and Modernity, Oxford, Clarendon Press, 1997, p. 8-36 («Law and society: the penetrative scheme and the juridical soul»).

21. Pour une critique de la conception atomiste et bourgeoise du XIX siècle et une proposition de constitutionnaliser plutôt une vision relationnelle du sujet de droit, voir J. Nedelsky, « Reconceiving rights as relationships », Review of Constitutional Studies, 1, 1993, p. 1-26. 
contre les atteintes inconstitutionnelles aux droits fondamentaux des citoyens, l'État lui-même étant soumis à la primauté d'un Droit qui garantit aussi bien la liberté des sujets que l'autorité légitime du souverain.

Les autres atteintes à la dignité humaine sont sociales. Elles prennent racine dans la culture des groupes, dans l'imposition à l'individu des normes et des stéréotypes collectifs. Le droit libéral, contrairement au droit absolutiste, n'entretient pas d'hostilité de principe envers l'autorité des «corps intermédiaires» et la gestion autonome de leurs affaires internes. Mais, il soumet la légitimité de leurs gestes d'autorité à l'exigence du consentement initial de chaque membre individuel et au respect de son droit de retrait ultérieur ${ }^{22}$. La liberté d'adhérer et le droit de circuler des individus sont les limites juridiques que le droit impose aux collectivités qui ne jouissent pas comme l'État des prérogatives de la contrainte inconditionnée. La notion même de «droit subjectif» implique que la décision de participer ou non à l'activité des groupes sociaux, de se soumettre aux exigences d'une fonction sociale, est laissée ultimement à l'arbitraire des volontés individuelles. Seuls les devoirs du citoyen envers l'État sont soustraits à la liberté de choisir des individus.

Dans l'épistémè politique de la démocratie libérale, la protection de la dignité humaine ne pose logiquement qu'un problème de fait et non un problème de droit. Pour que cette protection soit effective, il suffit de procurer aux citoyens des moyens suffisants pour défendre efficacement leurs droits constitutionnels contre l'État et pour exercer pleinement leurs droits civils contre l'autorité des groupes sociaux. La suprématie et la centralité d'un droit fondamentalement protecteur de la liberté individuelle font en sorte que la question de la dignité humaine se ramène à celle de l'accès pour tous à la justice et à l'égalité des chances.

La persistance des idées d' "accès à la justice» et d' «égalité des chances» dans le discours juridique et politique contemporain pourrait faire croire que nous en sommes encore là dans la longue marche en faveur du respect de la dignité humaine. Toute la difficulté se ramènerait à la disponibilité de ressources budgétaires suffisantes pour assurer l'accès aux tribunaux et à des mesures efficaces d'éducation populaire pour entretenir chez tous les citoyens la conscience de leurs droits et l'aspiration à les exercer librement. Cette vision du problème est pourtant erronée. Elle ne tient pas compte de l'avènement d'une seconde modernité dont la culture ne soutient plus la centralité et la primauté d'un droit imprégné de libéralisme individualiste. À ce stade, les obstacles à la protection de la dignité humaine deviennent juridiques autant sinon davantage que factuels.

\section{La seconde modernité ou l'épistémè économique du libéralisme corporatif}

Autant le XIX ${ }^{\mathrm{e}}$ siècle fut celui d'un droit d'inspiration politique instituant l'État comme garant de l'ordre social et de la sécurité des individus, autant le $\mathrm{XX}^{\mathrm{e}}$ siècle aura été celui d'un droit d'inspiration économique organisant le face-à-face des grandes personnes morales qui dominent la sphère du marché et de l'État providence qui pourvoit à la protection des individus contre les risques systémiques d'une société engagée dans une autotransformation constante et rapide.

La logique fondamentale de ce nouvel ordre des choses est moins politique qu'économique. La priorité n'est plus de construire un être-ensemble symbolique de tous les citoyens et d'organiser son fonctionnement démocratique. Il s'agit d'abord et avant tout d'aménager un régime fonctionnel d'interconnexion dynamique entre les organisations spécialisées et leurs stratégies respectives, pardelà la séparation formelle des domaines privé et public, en articulant aussi organiquement que possible les différents niveaux d'action et de régulation. Le défi premier est de maintenir l'unité du système général d'action et d'augmenter sa performance globale en misant au maximum sur la spécialisation fonctionnelle et l'intelligence stratégique des organisations considérées comme des acteurs de degré supérieur poursuivant prioritairement l'atteinte de leurs buts sectoriels. L'efficience du système économique, plutôt que l'autorité de l'État, et la liberté des entités organisationnelles, plutôt que celle des individus, deviennent les valeurs de référence qui inspirent l'évolution des normes sociales et du droit ${ }^{23}$.

La conscience sociale du basculement dans la seconde modernité devient chaque jour plus claire. Elle a progressé à la faveur d'idées générales comme celles d' "économie mixte», de "société juste» et de «concertation socioéconomique ». Elle progresse encore plus rapidement en ce début du XXI ${ }^{\mathrm{e}}$ siècle sous l'influence du discours prônant la «mondialisation de l'économie», «l'ouverture des frontières", «la libéralisation des échanges» et l'«harmonisation des normes» pour soutenir la performance systémique du «village global ${ }^{24}$.

Paradoxalement, la conscience du passage à la seconde modernité progresse peut-être plus lentement au sein de la communauté juridique que dans le reste de la société, malgré la transformation manifeste du droit positif tout au long du $\mathrm{XX}^{\mathrm{e}}$ siècle et plus rapidement encore depuis que la politique générale des États obéit aux manières de penser et d'agir promues par le néolibéralisme mondialisé. Les

22. S. J. Stoljar, «The internal affairs of associations", in Legal Personality and Political Pluralism, L. C. Webb (éd.), Cambridge, Cambridge University Press, 1958, p. 66-92. Aussi «Developments in the law. Judicial control of actions of private associations », Harvard Law Review, vol. 76, 1963, p. 983 sq. 23. P. Zumbansen et S. A. Ahmed, «The Transnational Law of Transitional Justice: a bridging exercise», CLPE Research Paper Series, 7, 4, 2008, p. 10: "Transnational regulatory fields are characterized by an amalgamation of public and private norm-generating actors that produce sets of norms and regulations, which escape the traditional framework of both public and private international law. » D. Mockle (dir.), Mondialisation et Etat de droit, Bruxelles, Bruylant, 2002. D. Mockle, La gouvernance, le droit et l'État. La question du droit dans la gouvernance publique, Bruxelles, Bruylant (Mondialisation et droit international), 2007.

24. Voir, par exemple, l'ouvrage collectif consacré à l'efficience des systèmes juridiques et à l'harmonisation transnationale des droits: J.-F. GaudreaultDesBiens, E. Kackaay, B. Moore et S. Rousseau (dir.), Convergence, concurrence et harmonisation des systèmes juridiques, Montréal, Thémis, 2009. 
juristes ont indéniablement pris acte de ces transformations juridiques et politiques sans lesquelles la seconde modernité n'aurait pu advenir. Mais ils l'ont fait en ordre dispersé, en formant des sous-communautés d'experts qui maîtrisent la connaissance très fine des règles de droit en vigueur dans leurs champs de spécialisation respectifs, mais qui ne prétendent pas à une connaissance adéquate des autres branches du droit ni à une compréhension unifiée de l'évolution d'ensemble du système juridique. Au-delà de leur domaine d'expertise dont ils défendent l'autonomie normative et institutionnelle, la plupart des juristes adhèrent encore à la théorie générale du droit de la première modernité, notamment à l'idée que le droit public reste gouverné par le principe de légalité et à l'idée que le droit privé s'édifie encore sur les règles fondamentales du droit commun classique ${ }^{25}$.

Il paraîtra prématuré pour cette raison de référer ici à une culture juridique de la seconde modernité dont les idées constitutives sont sous-estimées voire ignorées par la théorie générale du droit à laquelle adhèrent encore un grand nombre de juristes. Au risque de paraître prendre pour acquise ou d'exagérer la transformation paradigmatique en cours, il convient néanmoins de mettre en évidence les éléments principaux de la culture juridique émergente pour mieux comprendre le statut qu'elle réserve respectivement aux idées de pluralisme juridique et de dignité humaine.

Même si sa systématisation doctrinale reste à venir, la culture juridique de la seconde modernité est le produit de deux évolutions en cours depuis au moins un siècle. La première est celle de la désindividualisation croissante du traitement juridique des problèmes dans le droit de l'État comme dans les ordres juridiques partiels soumis à son pouvoir de surveillance et de contrôle. Aussi bien dans le droit social à travers lequel se construit l'État providence que dans le droit économique qui reconnaît le rôle moteur des organisations privées tout en les soumettant au pouvoir de réglementation d'instances publiques spécialisées, l'équilibrage juridique des rapports entre l'individuel et le collectif penche désormais en faveur du renforcement des pouvoirs organisationnels de détermination des objectifs à atteindre et de gestion des procédés pour y parvenir ${ }^{26}$.

Le droit des assurances sociales illustre fort bien dans la sphère des politiques publiques la faveur accordée à l'approche collective plutôt qu'individuelle des problèmes à prendre en charge et des solutions requises. Le traitement standardisé des réclamations d'indemnisation se substitue à l'ancienne approche judiciaire et individualiste du droit commun de la responsabilité civile. Le droit des collectivités locales évolue dans le même sens d'une rupture avec la philosophie libérale qui prévalait au $\mathrm{XIX}^{\mathrm{e}}$ siècle: les pouvoirs de réglementation des municipalités et de leurs instances spécialisées prennent le pas, au nom de l'ordre social ou de la gestion des services publics, sur l'ancienne protection scrupuleuse du droit individuel à la propriété privée. Même dans les diverses branches du droit privé s'observe le recul de la conception individualiste des droits et des pouvoirs au bénéfice d'une vision sociale, collective ou corporatiste. Dans le droit des sociétés par actions, et plus largement des personnes morales, s'affirment les prérogatives de gouvernance technocratique des dirigeants au détriment du pouvoir de blocage des actionnaires individuels ${ }^{27}$. En droit des contrats, la validité de principe des contrats collectifs ou des contrats types dont le contenu prévaut sur les volontés individuelles, est admise par le droit commun (validité du « contrat d'adhésion ») et souvent aménagée de façon privilégiée dans les branches spéciales du droit économique (notamment en droit du travail, en droit de la consommation, dans le droit des institutions financières). En droit des successions, l'exercice individuel de la liberté testamentaire est soumis au respect des règles de fond et de forme des régimes publics et privés d'assurance, de retraite, de Sécurité sociale, de protection du patrimoine familial.

Une même trame de fond traverse cette évolution générale du droit étatique au $\mathrm{XX}^{\mathrm{e}}$ siècle : l'individu conserve assez souvent la liberté juridique d'adhérer et de se soustraire aux stratégies d'action et aux programmes ou services offerts par les organisations publiques et privées (droits d'opting in et d'opting out), mais aussi longtemps qu'il y adhère, il perd juridiquement le droit d'initiative et de contrôle sur la détermination des buts et des moyens. Dans le droit du XX $\mathrm{XX}^{\mathrm{e}}$ siècle, le droit social des organisations dotées de la personnalité morale acquiert une préséance de principe sur la libre volonté individuelle, comme le grand capitalisme organisé s'impose au petit capitalisme indépendant dans la sphère du marché, comme les politiques publiques nationales s'imposent

25. Il faut toutefois convenir que ces deux idées du XIX siècle restent solennellement affirmées tout en haut de la pyramide des sources formelles du droit étatique. Dans le préambule de la Charte canadienne des droits et libertés, L.R.C. (1985), Ap. II, nº 44: «Attendu que le Canada est fondé sur des principes qui reconnaissent la suprématie de Dieu et la primauté du droit. » Dans la disposition préliminaire du Code civil du Québec, L.Q. 1991, c. 64: «Le Code est constitué d'un ensemble de règles qui, en toutes matières auxquelles se rapportent la lettre, l'esprit ou l'objet de ses dispositions, établit, en termes exprès ou de façon implicite, le droit commun. En ces matières, il constitue le fondement des autres lois qui peuvent elles-mêmes ajouter au Code ou y déroger. »

26. Si l'on prend à témoin le développement du droit administratif dans la sphère du droit public, le phénomène de désindividualisation s'exprime au $\mathrm{XX}^{\mathrm{e}}$ siècle par un pluralisme juridique intra-étatique, celui de la multiplicité des institutions administratives qui se substitue à l'ancien pluralisme juridique extra-étatique qui correspondait à la diversité des droits locaux jusqu'à la fin du XIX ${ }^{e}$ siècle. Telle est la thèse défendue avec beaucoup de force de conviction par H. W. Arthurs, Without the Law. Administrative Justice and Legal Pluralism in Nineteenth-Century England, Toronto, University of Toronto Press, 1985. En droit privé, si l'on prend à témoin l'évolution du droit des contrats, la «collectivisation » liée notamment à l'apparition des pouvoirs privés économiques et à la multiplication des contrats à effet collectif pourrait bien être «l'évolution juridique [...] la plus remarquable» selon G. Farjat, Droit privé de l'économie, 2, Théorie des obligations, Paris, PUF, 1975, p. 57-69 (66).

27. Après la forte poussée favorable au technocratisme managérial (A. A. Berle et G. C. Means, The Modern Corporation and Private Property, New York, Macmillan Press, 1939), le droit des sociétés par actions se préoccupe davantage aujourd'hui de la protection des actionnaires. Mais, ce regain de pouvoir profite aux actionnaires « institutionnels » et à leur domination stratégique. Les actionnaires individuels s'en trouvent juridiquement et économiquement soumis au contrôle des actionnaires institutionnels et à celui des dirigeants. 
aux volontés des collectivités locales et aux actes juridiques individuels dans la sphère du droit public.

La désindividualisation du droit libère ou désaliène le moi corporatif des personnes morales au détriment du moi individuel des personnes physiques. L'individu assuré, représenté, membre, employé, client, usager, mandataire ou même dirigeant se plie aux exigences du moi institutionnel de la personne morale. Il perd la qualité de sujet de droit jouissant de prérogatives originaires. L'exercice de ses droits économiques et sociaux obéit au régime des droits liés et finalisés par leur fonction sociale plutôt qu'à l'ancienne logique des droits subjectifs laissés à l'arbitraire des volontés individuelles. La soumission aux stratégies, programmes et règles des organisations est la contrepartie incontournable du droit individuel (entitlement) aux bénéfices qui dérivent de l'action collective.

Dans le nouveau contrat social du libéralisme corporatif, l'État lui-même n'est plus le sujet de droit souverain ni le seul « Sujet de l'histoire» (Hegel). Il partage son statut de grand ordonnateur du droit avec toutes les autres personnes morales auxquelles les citoyens-bénéficiaires se soumettent par servitude volontaire ou forcée: "Je consens, donc je suis. ${ }^{28}$ Les prérogatives juridiques que le libéralisme du XIX ${ }^{\mathrm{e}}$ siècle garantissait aux individus dans la sphère du droit privé, et même la plupart de leurs droits constitutionnels, sont désormais revendiqués en propre par chaque personne morale: «J'organise l'action collective, donc je dispose des pouvoirs juridiques que requiert la production des bénéfices attendus $»{ }^{29}$.

La déterritorialisation est la seconde évolution générale dont il faut prendre acte. La régulation juridique de l'action n'est plus soumise au strict respect des frontières et des juridictions formelles. L'ancienne doctrine légaliste de l'ultra vires recule au profit du leitmotiv d'un droit fonctionnel qui aménage la mise en réseau et la synergie de toutes les instances (locales, nationales et supranationales ; publiques et privées ; générales et particulières) qui peuvent et doivent contribuer au développement de l'économie, de la solidarité sociale, du savoir, de la santé, du divertissement, de la communication, de la culture... Les systèmes d'action et de régulation transfrontières peuvent légitimement se réclamer d'un droit fonctionnel dont l'effectivité requiert la participation et, par conséquent, l'autolimitation des droits territoriaux des États, des droits particularistes des personnes morales et des droits individuels des personnes physiques ${ }^{30}$.

La culture juridique encore diffuse qui inspire de façon souterraine cette évolution du droit positif s'alimente elle-même des idées exogènes qu'elle puise dans les sciences de l'organisation de l'action et de la régulation cybernétique des systèmes, dans les idéologies de la gestion participative et de l'interconnexion imposée par la nature systémique des choses, sinon par les exigences du bien commun: «Puisque nous sommes tous sur le même bateau, laissons de côté notre quant-à-soi formel et mettons nos ressources respectives au service de l'efficacité fonctionnelle générale ${ }^{31}$.

Quand on l'envisage plus particulièrement du point de vue de la citoyenneté liée à la territorialité de l'État dans la culture de la première modernité, on s'aperçoit que le nouveau fonctionnalisme juridique libère ou désaliène le moi enraciné dans l'appartenance nationale au profit d'un moi cosmopolite. Mais, ce moi déraciné n'est pas - ou du moins pas encore - le moi citoyen du monde promu, par exemple, par les militants altermondialistes qui s'opposent au néolibéralisme dominant. Le moi qu’il s'agit de libérer est plutôt le moi instrumental voué à la poursuite rationnelle et systématique d'un but commun.

Rien d'étonnant à cela puisque la culture générale de la seconde modernité ne repose plus sur une vision personnaliste et anthropomorphique du monde, celle qui convenait à une société d'individus, mais sur la métaphysique impersonnelle et technique qui convient à un univers de machines et à une société d'organisations. Comme ces dernières l'ont toujours réclamé de leurs membres, les individus sont requis de mettre au service du système général d'action le moi unidimensionnel qui maximisera les résultats des efforts collectifs et ultimement les bénéfices individuels.

Le nouveau fonctionnalisme juridique n'est pas la transposition de l'ancien idéal citoyen du niveau national au niveau supranational ou mondial. Il est plutôt la substitution d'un idéal cybernétique à l'ancien idéal humaniste. Le moi idéal de la seconde modernité n'est pas le moi multidimensionnel que les individus peuvent cultiver et défendre contre l'empiétement des pouvoirs organisés. Il est plutôt le moi unidimensionnel entretenu par la conscience d'appartenir à un système d'action et de régulation qui couvre désormais tous les niveaux et toutes les

28. M. Marzano, Je consens, donc je suis..., Paris, PUF, 2006, cité par M. Fabre-Magnan, « La dignité en droit : un axiome», p. 6.

29. Sur l'importance fondamentale du concept de "pouvoir», et sa distinction radicale avec la notion classique de «droit subjectif», puisque le pouvoir juridique permet à un acteur de droit privé, très souvent à une organisation dotée ou non de la personnalité morale, d'imposer une décision ou un acte juridique à autrui sans son consentement, voir E. Gaillard, Le pouvoir en droit privé, Paris, Economica, 1985. De nature organique lorsqu'ils découlent de la constitution interne d'une personne morale, les pouvoirs juridiques ont aussi très fréquemment une origine et une nature contractuelles. Voir P. Lokiec, Contrat et pouvoir. Essai sur les transformations du droit privé des rapports contractuels, Paris, LGDJ, 2004.

30. Sur la régulation pluraliste et fonctionnelle dans le droit transnational contemporain, voir notamment G. Teubner (éd.), Global Law Without a State, Dartmouth, Aldershot, 1997 ; G. A. Bermann, M. Herdegen et P. L. Lindseth (éd.), Transatlantic Regulatory Cooperation. Legal Problems and Political Prospects, Oxford, Oxford University Press, 2000 ; R. B. Hall et T. J. Biersteker (éd.), The Emergence of Private Authority in Global Governance, Cambridge, Cambridge University Press, 2002 ; C. Joerges, I.-J. Sand et G. Teubner (éd.), Transnational Governance and Constitutionalism, Oxford, Hart, 2004. Sur les difficultés de maintenir la cohérence et l'unité normative minimale du pluralisme juridique mondialisé, voir A. Fischer-Lescano et G. Teubner, «Regime-Collisions: the vain search for legal unity in the fragmentation of global law », Michigan Journal of International Law, 25, 2004, p. 999-1046.

31. C. Lafontaine, L'Empire cybernétique. Des machines à penser à la pensée machine, Paris, Seuil, 2004; P. Besson (dir.), Dedans-dehors. Les nouvelles frontières de l'organisation, Paris, Librairie Vuibert, 1997 ; É. Tassin, Un monde commun. Pour une cosmo-politique des conflits, Paris, Seuil, 2003. 
sphères de l'existence. Le moi idéal promu par le droit autant que par la cybernétique est un moi fondamentalement technocrate: plus savant que le profane car il sait comment fonctionne le système; plus fiable et loyal que le moi bourgeois du XIX ${ }^{\mathrm{e}}$ siècle, car il ne prétend pas contrôler les fins du système, mais seulement gérer ses procédés ou s'y soumettre efficacement. Ce moi idéal, on s'en doute puisqu'il vient de leur univers, est à la portée des personnes morales et conforme à leurs aspirations. Il n'est pas à la portée des personnes physiques et ne peut être entretenu par elles que sous le mode de l'aspiration aliénante ${ }^{32}$.

Précisons maintenant les représentations constitutives de la culture juridique de la seconde modernité, comme nous l'avons fait précédemment pour celle de la première modernité.

Comment l'État s'y conçoit-il? Non plus comme une entité abstraite, comme l'incarnation dans la personne morale par excellence de l'idée de «la société politiquement organisée». Non plus comme l'expression métaphysique de l'idéal démocratique entretenu par les citoyens, comme l' «État de droit». Plus prosaïquement et concrètement désormais, comme une vaste organisation bureaucratique et technocratique qui gère des biens collectifs, produit ou sous-traite des services, au bénéfice général de ses citoyens-usagers ${ }^{33}$.

Pour réaliser ses missions, l'organisation étatique a moins intérêt à se réclamer de son monopole symbolique de la souveraineté juridique qu'à entrer en rapport d'échange et de collaboration avec toute autre organisation contrôlant des ressources ou possédant une expertise qui peuvent lui être utiles. C'est ainsi qu'en théorie comme en pratique, l'État devient opérateur ou partie prenante d'une multitude de systèmes d'action et de régulation, en concertation avec les autres organisations qui ont atteint, dans chaque secteur d'intervention concerné et à chaque niveau opérationnel pertinent, la qualité organisationnelle et l'importance stratégique qui leur permettent de se poser en interlocutrices incontournables et en partenaires souhaitables de la puissance publique. Les autres États de la communauté internationale, les grandes entreprises de l'économie nationale et mondiale, les ONG de la société civile supranationale et les associations sans but lucratif du pays, toutes ces personnes morales ont dès lors une vocation privilégiée à jouer un rôle significatif dans le système d'action et de régulation que l'État aménage lui-même ou auquel il adhère à la faveur d'un calcul pragmatique, sans se considérer menotté par l'ancienne mystique dogmatique d'un État souverain qui peut tout et ne négocie avec personne.

L'État se conçoit lui-même, désormais, en rapport d'interconnexion nécessaire ou utile avec les autres organisations de son environnement. La préoccupation première n'est plus de mobiliser son pouvoir législatif d'édicter le droit d'application générale ni le pouvoir judiciaire d'en imposer le respect, mais le pouvoir exécutif d'orienter son comportement stratégique selon l'intérêt bien compris des citoyens, par une politique générale de coopération interorganisationnelle. L'atteinte des objectifs stratégiques impose de mettre en veilleuse la représentation politique de l'unité de l'État par-delà la pluralité sociale ( $E$ Pluribus Unum») pour mettre au premier plan la représentation économique du pluralisme organisationnel intra-étatique et extra-étatique qu'il s'agit de mobiliser dans le meilleur intérêt des citoyens («One Among Many») ${ }^{34}$.

Puisqu'il s'agit d'abord et avant tout d'insérer l'État au centre ou à la périphérie du pluralisme organisationnel qui prévaut de facto dans chaque secteur d'intervention, la conception de la juridicité adéquate à la société des organisations ne peut se transformer elle-même que dans le sens du pluralisme juridique. Le paradigme du droit conforme à l'idéal de la seconde modernité n'est plus celui de la pyramide homogène et hiérarchisée, mais celui du réseau hétérogène et fonctionnel ${ }^{35}$. L'idéal type de l'ordre juridique à mettre en place et à faire fonctionner efficacement est celui de l'entreprise multinationale, de l'organisation à vocation transnationale ou, plus abstraitement, du système d'action, de coordination et de régulation d'envergure mondiale.

Le droit idéal est celui qui tout à la fois reconnaît, protège et mobilise la diversité des acteurs et des parties prenantes. C'est un droit d'intégration fonctionnelle, différencié en plusieurs niveaux et rayons de fonctionnement, qui gère dynamiquement l'interconnexion de multiples unités opérationnelles plus ou moins autonomes

33. Le passage de l'ancienne métaphysique d'un État unifié sur la base des principes généraux du droit public à la nouvelle culture du fonctionnalisme juridique se réclamant des avantages de la spécialisation organisationnelle se remarque clairement dans l'évolution du droit du contrôle judiciaire exercé par les tribunaux de droit commun sur les décisions et les interprétations juridiques des multiples tribunaux administratifs dont la création au $\mathrm{XX}^{\mathrm{e}}$ siècle a rendu beaucoup plus complexe l'administration de la justice étatique. On trouvera une synthèse saisissante de cette évolution en droit administratif canadien dans l'ouvrage de S. Comtois, Vers la primauté de l'approche pragmatique et fonctionnelle. Précis du contrôle judiciaire des décisions de fond rendues par les organes administratifs, Cowansville, Éditions Yvon Blais, 2003. Comme l'auteure le souligne, cette évolution ne va pas sans une certaine ambivalence. Le recul de l'ancienne doctrine légaliste de l'ultra vires fondée sur une conception hiérarchique du contrôle judiciaire s'effectue au profit d'une politique de déférence des cours supérieures envers l'expertise des tribunaux spécialisés. Mais, en parallèle, les cours de droit commun se montrent plus ouvertes qu'auparavant à contrôler la rationalité et la légitimité des décisions du pouvoir exécutif. Liée étroitement à la politique constitutionnelle de protection des droits et libertés des individus contre les interventions intrusives d'un pouvoir exécutif omniprésent, cette seconde évolution suggère que la dynamique intraorganisationnelle de l'État contemporain pourrait se considérer comme la manifestation d'un mono-pluralisme juridique où se reconnaît une version nouvelle et plus complexe de la dialectique entre l'unité et la diversité.

34. Sur la nouvelle culture étatique dont se réclament les détenteurs des pouvoirs exécutifs pour opérer une véritable « dénationalisation » de l'ordre juridique interne en faveur d'une ouverture fonctionnelle aux normes et pratiques de la «bonne » gouvernance transnationale, voir S. Sassen, « The state and globalization ", in The Emergence of Private Authority in Global Governance, p. 91-112.

35. K. Benyekhlef, Une possible histoire de la norme, Montréal, Thémis, 2008. F. Ost et M. van de Kerchove, De la pyramide au réseau? Pour une théorie dialectique du droit, Bruxelles, Publications des Facultés universitaires Saint-Louis, 2002. 
selon les exigences de leurs fonctions respectives. C'est un droit composite et flou, tantôt formel tantôt informel, rigide ou souple, en remaniement constant à la faveur des ajustements souhaitables dans les rapports entre le système d'action et son environnement. Dans chaque régime sectoriel et dans la configuration générale des interactions entre les divers systèmes d'action, le pluralisme juridique évolue en comptant sur la valeur normative des pratiques efficaces (best practices) autant que sur celle des règles établies, en respectant les autonomies utiles, mais en surplombant les frontières qui nuisent à l'efficacité.

Dans les sphères autrefois étanches mais désormais poreuses du droit public et du droit privé, du droit national et du droit supranational, de l'action politique et de l'action économique, le nouvel idéal de la juridicité s'incarne dans l'harmonisation dynamique des droits ouverts les uns aux autres plutôt qu'enfermés dans leur seule logique interne. Dans tous les secteurs et dans toutes les cultures juridiques partielles s'effectue le passage du droit impérial au droit-ressource, s'insinue l'idée primordiale que la juridicité se porte comme un vêtement de course et non comme un obstacle qui limite la performance ${ }^{36}$.

Dans la culture juridique de la seconde modernité, il en va de la conception de la personne comme de celle du droit lui-même. L'être humain ne se conçoit plus comme l'unité irréductible et inviolable d'un corps et d'une âme, mais comme un ensemble décomposable et mobilisable de ressources physiques, psychologiques et intellectuelles potentiellement utiles voire nécessaires à l'efficacité des systèmes d'action et de régulation. Selon les besoins fonctionnels, le corps peut être ici ou là, l'âme peut être ailleurs et l'esprit peut être nulle part.

Le face-à-face paradigmatique n'est plus celui du citoyen devant l'État ou du sujet de droit en interaction avec un autre sujet de droit, mais le face-à-face entre l'être humain et la machine ou la technologie dont il faut apprendre à se servir pour mieux la servir. Le surveillant d'un robot industriel, le pilote d'un avion supersonique, l'internaute branché à son ordinateur ou le personnage virtuellement exposé dans la galerie de portraits de Facebook ou de Twitter, ne sont plus un corps gouverné par son âme singulière, mais une ressource individuelle mise au service d'une machine animée par la logique d'un système organisationnel, mais une subjectivité d'emprunt mise en scène par un système de représentation transpersonnelle.

Le corps de la personne postmoderne fait corps avec celui de la machine. Son âme fait système avec l'âme de l'organisation et du réseau. La dissociation et la démultiplication du moi individuel font écho à la diversité et à la multiplicité des systèmes qui le mobilisent. La personne n'a plus de subjectivité forte et unifiée car l'atteinte du but poursuivi ou la jouissance du fantasme entretenu n'est possible qu'à travers la symbiose de l'être humain et de la machine. La pensée, la volonté et l'affectivité singulières de l'individu sont disqualifiées par la complexité du système, par l'opacité et la rapidité de ses opérations. Le moi se maintient en relation physique et symbolique avec des choses et des technologies qui ne peuvent lui renvoyer qu'une image matérialiste ou technique de sa personne. La machine, l'organisation, le système, ne sont plus l'outil qui prolonge la main ou la pensée de l'être humain. Le corps, l'âme, la personnalité de l'individu, sont plutôt le prolongement de la machine, de la rationalité instrumentale de l'organisation et de l'impersonnalité du système ${ }^{37}$.

Quelle est désormais la représentation dominante de la personnalité juridique? Elle n'est plus la valeur ajoutée que procure d'emblée et irrévocablement, à chaque personne physique, l'appartenance symbolique au grand tout d'un Droit produit mythiquement par et pour l'être humain. Elle est plutôt la ressource juridique rendue disponible, facultativement et pour un temps discrétionnaire, à toute entité (un être humain, un animal, un groupement, une entreprise, une organisation, un système, une chose, une idée, une aspiration, un sentiment...) dont on trouvera opportun qu'elle prenne la forme ou le masque symbolique d'une personne aux yeux d'un ou de plusieurs systèmes de droit qui se conçoivent eux-mêmes comme des pourvoyeurs de ressources utiles aux opérations des autres systèmes d'action et de régulation.

La personnalité juridique caractéristique de la seconde modernité est celle de la personne morale: un statut juridique facultatif, substituable, à géométrie variable, révisable, cumulable, dont l'attribution et la dissolution peuvent être décidées par autrui. Songeons, par exemple, à la personnalité juridique qu'aura ou n'aura pas la filiale d'une entreprise multinationale opérant dans telle ou telle juridiction nationale. La jouissance de la personnalité morale n'est pas une qualité essentielle, mais seulement accidentelle ou mieux circonstancielle, de l'entité considérée. Elle advient ou cesse à la faveur d'un calcul d'utilité et souvent à l'issue d'un rapport de forces entre les joueurs intéressés. Elle n'a plus l'automatisme d'un a priori dogmatique, d'une nécessité

36. L’analyse économique du droit a joué un rôle prépondérant dans cette évolution de la culture juridique en mettant au premier plan l'idée que les institutions juridiques doivent s'analyser du point de vue de la performance des acteurs-consommateurs, comme des produits offrant des ressources et imposant des contraintes justiciables du seul critère décisif de l'efficience économique, des produits normatifs qu'il convient pour cette raison de soumettre au jeu d'un marché d'envergure transnationale au sein duquel les acteurs peuvent librement choisir les arrangements normatifs les plus attractifs. Les rapports Doing Business publiés par la Banque mondiale depuis 2004 sont l'instrumentalisation la plus contestable de cette idéologie. Voir, à leur sujet, B. Du Marais (dir.), Des indicateurs pour mesurer le droit? Les limites méthodologiques des rapports Doing Business. Études du programme de recherches sur l'attractivité économique du droit, Paris, La Documentation française, 2006. Pour une version plus académique mais non moins réductrice, voir R. Romano, « Law as product: some pieces of the incorporation puzzle », Journal of Law, Economics \& Organization, 1, 1985, p. 225.

37. Sur le décentrement et l'instrumentalisation de l'être humain dans les théories de la cybernétique, de la communication, du structuralisme, du systémisme et du postmodernisme qui se sont disputé la faveur des scientifiques et des intellectuels depuis le milieu du XX siècle, voir la synthèse pénétrante de C. Lafontaine, L'Empire cybernétique... Le titre d'un ouvrage du père de la cybernétique est candidement expressif: N. Wiener, Cybernétique et société. L'usage humain des êtres humains, P.-Y. Mistoulon (trad.), Paris, UGE, 1954. 
métaphysique, comme c'était le cas dans la culture juridique anthropomorphique de la première modernité.

La personnalité juridique, au premier chef celle des personnes physiques, se concevait, dans une perspective humaniste, comme l'attribut nécessaire d'un être de besoins qui jouira de sa qualité de sujet de droit pour garantir juridiquement la satisfaction de ses besoins, la protection de ses intérêts. La personnalité juridique des personnes morales se conçoit plutôt, dans une perspective technique, comme l'appendice technologique qu'on ajoute ou non à une entité conçue comme une ressource ou un ensemble de ressources, selon les besoins du système qui mobilise cette entité qui n'a pas d'autres besoins que celui qu'on lui fournisse les aliments nécessaires à son fonctionnement utile et préférablement optimal ${ }^{38}$.

La culture juridique de la seconde modernité opère un renversement de valeur paradigmatique entre les personnalités juridiques de l'être humain et de la personne morale. Dans la culture classique, la personnalité juridique de l'être humain s'imposait la première à l'esprit juridique. Elle servait de modèle de référence pour la conception de la personnalité juridique des autres entités, précisément désignées comme "personnes", mais seulement «morales» ou «légales» (legal persons) puisque leur existence ne pouvait tenir à la possession d'un corps humain, mais provenait de la reconnaissance juridique de la valeur morale qu'elles incarnaient ou plus prosaïquement découlait d'une fiction créée par la loi étatique $^{39}$. Dans la nouvelle culture juridique, c'est plutôt la personnalité juridique de la personne morale qui advient la première et sert de modèle pour une vision renouvelée de la personnalité juridique des êtres humains. Le jour n'est peut-être pas si éloigné où l'ancien «droit des personnes » subdivisé en "droit des êtres humains» et «droit des personnes morales » sera remplacé dans la dogmatique juridique par le «droit des entités juridiques » subdivisé en "droit des entités organisationnelles» et «droit des entités humaines». Autant la première dogmatique fut gouvernée par l'idée de «personne», autant la seconde le sera par l'idée de «fonction ${ }^{40}$.

Ce n'est pas ici le lieu de récapituler le glissement qui s'est opéré de l'idée fondamentale de " personne » à celle de «fonction» dans le droit positif et dans la théorie générale de la personnalité juridique. Pour les besoins de notre réflexion présente, il convient plutôt de préciser ce qui change fondamentalement en droit des personnes dans une culture juridique dominée par le paradigme du pluralisme juridique et ce qu'il advient, en conséquence, de l'idée de dignité humaine lorsqu'on la transpose dans l'univers conceptuel d'un droit général des entités juridiques répondant prioritairement aux besoins d'une civilisation matérialiste et technique.
Au stade de la première modernité, l'octroi de la personnalité juridique faisait partie des attributions détenues par l'État de façon monopoliste. C'était, parmi d'autres, un pouvoir exclusif relevant de sa souveraineté juridique. L'exercice de ce pouvoir fut d'abord absolutiste, autoritaire et discrétionnaire: n'accédaient au statut de personne juridique que l'entité organisationnelle jugée utile à la poursuite des buts de l'État, que l'être humain jugé digne de produire par lui-même et pour lui-même des effets de droit. À la faveur des idéologies libérale et démocratique, l'exercice de ce pouvoir se plia lui-même de plus en plus, en ce domaine comme en d'autres, aux valeurs de liberté et d'égalité: deviendront personnes morales toutes les entités qui choisiront d'adopter ce statut pour autant qu'elles respecteront les exigences formelles des lois d'incorporation générale; seront personnes juridiques tous les êtres humains dès lors qu'ils auront satisfait aux exigences de la viabilité biologique.

L'émergence de la culture juridique pluraliste et technicienne permet d'entrevoir une troisième évolution fondamentale dans l'exercice du pouvoir d'attribuer la personnalité juridique. Même d'un strict point de vue formaliste, ce pouvoir ne sera plus exercé exclusivement par l'État, mais partagé avec d'autres organisations habilitantes. C'est déjà le cas lorsqu'un État reconnaît en fait sinon en droit la personnalité juridique conférée par un autre État à l'entité organisationnelle qui est venue agir et investir sur son territoire ${ }^{41}$. Ce sera de plus en plus souvent le cas lorsque l'État tiendra pour personnes morales les entités créées par ses interlocuteurs et partenaires, soit en vertu d'une délégation formelle de l'État lui-même, soit même de façon indépendante parce qu'à toutes fins utiles la reconnaissance de la personnalité morale conférée privément s'énonce comme une condition imposée par l'instance dont l'État recherche activement la collaboration. Le paradigme pluraliste de la juridicité commande logiquement et pratiquement l'attribution pluraliste de la personnalité juridique.

En aménageant de façon plurielle le pouvoir d'attribuer la personnalité juridique, le droit de la seconde modernité ouvre tout grand la porte à une nouvelle façon de concevoir le statut qu'il s'agit d'attribuer ou de refuser aux entités intéressées. En écho à la culture juridique générale, la nouvelle conception sera économique plutôt que politique, technique plutôt qu'humaniste. Quand il exerçait son monopole sur la création des personnes morales, l'État concevait leur statut juridique à sa propre image: chaque personne morale serait comme lui, mais en plus petit, un corps politique unifié et indépendant, jouissant d'une volonté juridique libre et obéissant, dans son fonctionnement interne, à l'éthique d'une démocratie représentative au sein de laquelle les dirigeants

38. Sur la technicité du concept de «personne morale» dans un droit positif qui a pris congé des fondements philosophique et politique de l'ancienne pensée juridique anthropomorphique, voir C. Bouchard, La personnalité morale démythifiée. Contribution à la définition de la nature juridique des sociétés de personnes québécoises, Québec, Presses de l’Université Laval, 1997, p. 127 sq.

39. T. Baty, «The rights of ideas - and of corporations (1919-1920)», Harvard Law Review, vol. 33, 1963, p. 358 sq.

40. R. Kraakman et al., The Anatomy of Corporate Law. A Comparative and Functional Approach, Oxford, Oxford University Press, 2004

41. Voir C. Day Wallace, The Multinational Enterprise and Legal Control. Host State Sovereignty in an Era of Economic Globalization, The Hague, Martinus Nijhoff Pub., 2002, p. 101-158. P. Muchlinski, Multinational Enterprises and the Law, Oxford, Blackwell, 1995, p. 57-120. 
doivent périodiquement répondre de l'exercice de leurs pouvoirs délégués devant l'assemblée générale des membres, actionnaires, cotisants, coopérants. Quand elles auront affirmé solidement leur détention légitime du pouvoir d'attribuer la personnalité juridique, les organisations imposeront leur propre manière de concevoir la personnalité d'une entité. À leur image, l'entité sera personnifiée en relation exclusive avec le but qui lui est assigné au sein d'un système plus grand qu'elle-même. L'entité fonctionnelle ainsi reconnue dans la spécificité de sa place au sein d'un ensemble ne se concevra pas dans l'indépendance de son existence propre, mais dans son interdépendance avec les autres composantes du système de référence auquel elle se trouve liée organiquement. Moins qu'une personne libre en interaction volontaire avec d'autres personnes libres, elle sera l'équivalent d'un maillon inséré dans une chaîne, d'une case dessinée dans la ligne d'autorité ou parmi les divisions fonctionnelles d'un organigramme, d'un sous-programme opérant à l'intérieur d'un programme informatique. Moins qu'une personne juridique égale à toute autre personne juridique, elle jouira d'un statut taillé sur mesure pour l'exercice efficace de sa fonction: ses compétences, ses pouvoirs, l'ampleur de sa liberté d'action et de sa discrétion décisionnelle, sa structure interne démocratique ou autocratique selon les besoins, tout cela composera une personnalité spécifique et contingente plutôt que gouvernée par l'adéquation à un modèle uniforme et égalitaire de la personnalité juridique.

Variable en quantité et en qualité, provisoire ou durable, retirée ou refusée selon les possibilités de lui procurer les mêmes attributions fonctionnelles par des techniques de substitution empruntées au droit des biens, au droit des contrats ou au droit des organisations non personnifiées, égale ou inégale à celle des autres entités, la personnalité juridique sera dès son origine et ne cessera pas d'évoluer ensuite en relation directe au système organisationnel de référence, sera fonction, du début à la fin, des besoins de ce système plutôt que de ses besoins propres. Comme la machine qui doit son existence au projet d'autrui et reste sous le contrôle d'une volonté étrangère à la sienne.

Dans la société des organisations et des systèmes d'action, dans la culture de la régulation cybernétique, on ne se préoccupe plus de faire naitre juridiquement des entités citoyennes de la société politique ou agentes créatrices de la société civile, mais des entités qui pourront et devront se comporter comme des rouages utiles au fonctionnement de leurs systèmes d'appartenance respectifs. Songeons encore ici au statut exemplaire d'une filiale intégrée à un groupe multinational d'entreprises : sa personnalité est décidée par une autre personne, la société mère ; ses décisions politiques et stratégiques sont contrôlées statutairement, contractuellement ou de facto par des instances externes; ses compétences, ses pouvoirs, son autonomie réelle dépendent de la dynamique interne et du positionnement stratégique d'un groupe macroorganisationnel qui préside aux destinées d'un nombre souvent considérable de personnes morales intégrées en son sein et contrôlées directement ou non par un centre décisionnel qui peut fort bien lui-même ne pas avoir de personnalité juridique, mais qui détient aux yeux de tous les clés maîtresses du système général et les leviers de commande ultimes des opérations ${ }^{42}$.

Qu'advient-il, en droit et en fait, de la dignité des êtres humains dans cet univers dominé par les organisations? Puisque la logique générale de l'ordre juridique est celle d'un droit fonctionnel, puisque la personnalité morale est l'archétype de toute personnalité juridique, le statut des êtres humains ne peut être qu'à l'image de celui de toutes les autres entités reconnues: au mieux celui d'une fonction plus ou moins élevée et valorisée, parfois celui d'un rouage plus ou moins central, souvent celui d'un simple pion sur l'échiquier. La dignité attendue de chaque être humain consistera à se tenir à la hauteur de la fonction, de l'opération ou du rôle subalterne voire sacrificiel qui lui revient à l'intérieur du système d'appartenance ou plutôt des multiples systèmes qui lui assignent une place. Si tel ou tel système d'action ne lui offre en son sein aucune place, ou si l'être humain s'exclut luimême d'un système qui s'offre à lui, l'individu peut encore prétendre à une existence biologique et psychologique, à une existence sociale en marge des organisations, mais il n'a pas accès à la personnalité juridique qui, seule, compte désormais et n'a plus, en conséquence, d'aspiration juridiquement protégée à la dignité. La dignité humaine, comme la dignité d'une machine ou de toute autre entité, ne se conçoit plus et n'est plus reconnue sans la subordination nécessaire à l'autorité et aux règles de fonctionnement des systèmes dont l'existence est la seule qui soit désormais indispensable à la vie de la société organisationnelle et aux yeux de son droit pluriel.

Dans la seconde modernité parvenue à maturité, les atteintes à la dignité humaine ne seront pas d'une autre nature ou d'une plus grande gravité que les atteintes à la dignité de toute autre entité reconnue. Ce seront des atteintes physiques ou symboliques à la fonction, au rôle ou à la capacité technique d'une composante systémique plutôt que d'un être à part entière. Exclure ou détruire un être humain ne portera pas plus ni moins à conséquence, juridiquement et socialement, que l'exclusion ou la destruction d'une chose utile, d'une machine performante ou d'une idée rentable. Chaque entité, humaine ou non, ne pourra prétendre être respectée que dans son droit fondamental à l'utilité ${ }^{43}$.

42. Pour une théorisation de l'entreprise multinationale comme réseau interorganisationnel transnational, voir S. Ghoshal et D. E. Westney (éd.), Organization Theory and the Multinational Corporation, New York, Palgrave, 2005. Pour une analyse juridique du bouleversement induit dans le droit de la personnalité morale par le phénomène contemporain des entreprises transnationales, voir P. I. Blumberg, The Multinational Challenge to Corporation Law. The Search for a New Corporate Personality, New York, Oxford University Press, 1993 ; J. E. Antunes, Liability of Corporate Groups. Autonomy and Control in Parent-Subsidiary Relationships in US, German and EU Law, Deventer, Kluwer, 1994.

43. Sous ce paradigme de l'utilité, il n'y a plus de raison impérieuse de limiter l'attribution de la personnalité juridique aux êtres humains et à leurs divers types de regroupements. L'élargissement de cette attribution à des entités non humaines, notamment à des instruments purement techniques de 


\section{Pour une troisième modernité pluraliste et humaniste}

La partie n'étant pas encore jouée définitivement, comment peut-on imaginer un renversement de tendance faisant en sorte que les idées contemporaines de pluralisme juridique et de dignité humaine se renforcent mutuellement plutôt que de s'opposer? Cet exercice d'imagination, à contre-courant du libéralisme corporatif et de l'épistémè technique qui dominent aujourd'hui, n'a pas à s'excuser de son caractère forcément utopique. Il prétendra néanmoins convaincre utilement en se réclamant, d'une part, d'un réalisme social nécessaire, puisqu'il faut d'abord réaliser pleinement le tragique de la situation présente, d'autre part, d'un idéalisme juridique ancré dans l'expérience de la modernité, puisqu'il veut rêver la solution adéquate en demeurant fidèle au principe constitutionnel en vertu duquel les droits subjectifs fondamentaux des individus doivent prévaloir sur les pouvoirs juridiques des organisations chaque fois que l'exigence d'atteinte minimale et proportionnelle aux premiers a été outrepassée par une pratique unilatéralement inspirée par la recherche de l'efficacité organisationnelle ${ }^{44}$.

Réalisons d'abord le tragique de la situation en recon-naissant que le triomphe théorique et pratique de l'idée de pluralisme juridique s'effectue aujourd'hui au prix d'une conception aliénée de la dignité humaine ramenée à un commun dénominateur technique plutôt qu'humaniste. La vision des choses exposée dans la section précédente emporte-t-elle la conviction que telle est bien la réalité profonde, la vérité factuelle, de la situation présente? Admettons, pour les fins de la réflexion, qu'on puisse encore en douter raisonnablement et que la lecture des faits est commandée en dernière instance par le penchant optimiste ou pessimiste du lecteur, que leur interprétation reste dépendante de l'émerveillement ou du désarroi de l'interprète devant la puissance magique ou funeste des techniques qui participent désormais du monde vécu.

Plutôt que de nous réclamer d'une impossible vérité unilatérale des faits, faisons reposer notre réflexion sur le postulat d'une égale vraisemblance des lectures contradictoires et considérons les enjeux de la situation présente pour déterminer s'il vaut mieux réfléchir à partir de l'hypothèse du meilleur ou du pire. La seconde option nous paraît, à cet égard, s'imposer au nom de la prudence, de la sagesse ou, en d'autres termes, d'un principe de précaution dicté par la gravité et l'irréversibilité des atteintes qui seront imposées à la dignité humaine si l'accroissement de la puissance organisationnelle n'est pas contré par une protection juridique plus étendue et plus rigoureuse des droits fondamentaux de l'être humain.

Le risque ultime de la tendance contemporaine est d'aboutir, d'une part, à un terrorisme organisationnel qui laisse l'individu devant le choix entre l'inclusion aliénante et l'exclusion marginalisante ${ }^{45}$, de conduire, d'autre part, à un totalitarisme systémique qui condamne les personnes les plus dévouées à leurs multiples fonctions à subir dans leur corps et dans leur âme l'impossible réconciliation des demandes que chaque organisation ou chaque sous-système rend chaque jour plus exigeantes, sans s'inquiéter des limites physiques et psychiques de l'individu qui en est le destinataire commun ${ }^{46}$. Dans le langage utilisé dès 1943 par Simone Weil, le risque ultime du pluralisme juridique contemporain est la négation radicale du besoin de responsabilité de l'être humain et de son besoin d'ordre ${ }^{47}$.

Est-il réaliste de croire que le droit contemporain, avec ses solutions anciennes et nouvelles, offre un rempart suffisant contre l'avènement de ce risque ultime de la société des organisations? La jurisprudence des juridictions nationales et supranationales fournit des exemples probablement plus nombreux aujourd'hui et certainement plus médiatisés d'invocations victorieuses des droits de la personne pour invalider les règles ou les pratiques des organisations. L'idée de constitutionnaliser le fonctionnement interne des organisations privées autant que publiques, transnationales autant que nationales, à but lucratif autant que caritatif, sur le modèle de la démocratie libérale progresse en théorie générale du droit $^{48}$. Le sentiment se répand que la dynamique même de la mondialisation économique mènera tôt ou tard à la construction d'un ordre juridique mondial instituant une sorte de mono-pluralisme fédéral d'envergure planétaire au sein duquel pourrait être affirmée, face au gouvernement mondial et à tous les gouvernements nationaux, la valeur éminente d'un humanisme cosmopolite ${ }^{49}$.

communication, s'impose dès lors que ces entités peuvent gagner en utilité fonctionnelle si elles se voient conférer les attributs de la personnalité juridique. Voir G. Teubner, «Rights of non-humans? Electronic agents and animals as new actors in politics and law », Journal of Law and Society, 33, 2006, p. 497. 44. Le critère d'atteinte minimale et proportionnelle a notamment été retenu par la Cour suprême du Canada pour déterminer si une règle de droit qui porte atteinte à une liberté fondamentale peut malgré cela être considérée valide parce qu'elle s'avère en deçà « des limites qui soient raisonnables et dont la justification puisse se démontrer dans le cadre d'une société libre et démocratique » (Charte canadienne des droits et libertés, L.R.C. (1985), Ap. II, $\mathrm{n}^{\circ}$ 44, art. 1). Ce critère trouve son origine dans l'arrêt R. c. Oakes, [1986] 1 R.C.S. 103.

45. J.-G. Belley, "Gouvernance et démocratie dans la société neuronale», in La démocratie à l'épreuve de la gouvernance, L. Cardinal et C. Andrew (éd.), Ottawa, Les Presses de l'Université d'Ottawa, 2001, p. 153-171 (165-169).

46. V. de Gaulejac, La société malade de la gestion: idéologie gestionnaire, pouvoir managérial et harcèlement social, Paris, Seuil, 2005. Aussi excessive qu'elle puisse paraître en théorie, la thèse du totalitarisme et du terrorisme organisationnels trouve d'ores et déjà des manifestations empiriques dans l'actua-

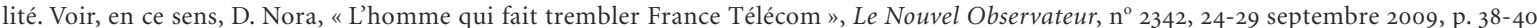
(24 suicides chez les employés de cette société depuis le début de l'année 2008; 22 suicides en 2003 et 29 en 2002...).

47. S. Weil, L'Enracinement. Prélude à une déclaration des devoirs envers l'être humain, Paris, Gallimard, 1949, p. 25 («besoin vital d'initiative et de responsabilité») et p. 18 ( «que nul ne soit contraint de violer des obligations rigoureuses pour exécuter d'autres obligations» - besoin d'ordre).

48. C. Joerges, I.-J. Sand et G. Teubner (éd.), Transnational Governance and Constitutionalism; C. D. Stone, «Corporate vices and corporate virtues: do public / private distinction matters? ", Pennsylvania Law Review, vol. 130, 1982, p. 1441 sq.

49. É. Tassin, Un monde commun... ; I. Ramonet, Géopolitique du chaos, Paris, Gallimard, 1999. 
Mais, la vraisemblance historique et sociologique suggère, à notre avis, que ces progrès actuels et prévisibles de la conscience juridique contemporaine participent eux-mêmes, sous le mode de la résistance ou de l'anticipation, de la montée en puissance et de l'hégémonie des organisations au cours des deux derniers siècles et dans toutes les sociétés de la modernité avancée. La sagesse nous enjoint de reconnaître que les principes de respect des libertés individuelles, de la démocratie et de l'humanisme ne sont et ne pourront être que des principes juridiques correcteurs et non directeurs aussi longtemps que l'évolution de l'économie, de la politique et de la vie sociale continuera d'être commandée par la logique première de la croissance matérielle et par l'impératif des moyens technologiques pour y parvenir. Cette logique et cet impératif placent les organisations et systèmes à l'avant-garde de l'action, bien au-devant et bien au-dessus des individus. Comme Julien Benda le constatait déjà il y a plusieurs décennies, les principes de la démocratie libérale qui protègent la personne ne peuvent être pratiqués qu'en second et seront souvent jugés dysfonctionnels dans une civilisation qui met ses espoirs de progrès dans les dividendes que procure la puissance organisationnelle. La primauté du principe humain sur le principe fonctionnel n'est envisageable qu'au prix d'une option ferme en faveur de la liberté de l'individu et de la démocratie, même lorsqu'elle fait obstacle à l'efficacité générale ${ }^{50}$.

Pour que cette option soit envisageable et crédible, encore faut-il identifier le principe directeur qui présidera à la solution des conflits entre la promotion du pluralisme juridique, dont les organisations sont les premières bénéficiaires, et le respect de la dignité humaine, au profit des seuls êtres humains. Encore faut-il conférer à ce principe prééminent toute l'extension et toute la rigueur que requiert le tragique de la situation présente. Quel principe, de quelle portée, peut-on imaginer au centre et au sommet de l'ordre juridique pluraliste et humaniste dont nous rêverons l'avènement au cours d'une troisième modernité utopique?

Pour faire contrepoids et s'imposer au droit de l'utilité, de l'efficacité et de l'efficience dont se réclament les organisations, nous imaginerons le droit à la vie que les citoyens des démocraties peuvent déjà invoquer constitutionnellement, mais en lui conférant toute la portée que requiert la gravité des enjeux contemporains ${ }^{51}$. Nous retiendrons au profit du droit fondamental à la vie les significations et dimensions que lui ont déjà reconnues les droits positifs nationaux et supranationaux les plus sensibles à la rhétorique et à l'éthique humanistes.

Le premier de ces attributs juridiques, que nous retiendrons à titre de postulat, tient dans l'affirmation que ce droit fondamental à la vie, contrairement à la plupart des autres droits constitutionnels modernes, ne peut être revendiqué que par les êtres humains et ne saurait l'être en propre par les personnes morales ${ }^{52}$. Il y a là le principe clair et net d'une discrimination juridique on ne peut plus pertinente puisque les seules victimes de l'exclusion seront les entités organisationnelles dont il s'agit précisément de contrer la puissance ${ }^{53}$.

Nous ajouterons à ce postulat les dimensions que les droits positifs modernes ont déjà consacrées de façon au moins déclaratoire sinon opérationnelle : le droit à la vie tout court qui protège l'existence de tout être humain contre la peine capitale, même celle qu'on prétendrait justifier au nom d'une justice immanente ou de l'intérêt

50. J. Benda, La grande épreuve des démocraties. Essai sur les principes démocratiques, New York, Éditions de la Maison Française, 1942 : «La démocratie, en tant qu'elle tient pour valeur suprême, non pas l'organisation mais la liberté de la personne - condition cruciale de sa dignité - pose bien la question de savoir si, en la repoussant ou en l'adoptant, l'humanité se veut puissante ou morale.» (p. 211).

51. «Chacun a droit à la vie, à la liberté et à la sécurité de sa personne ; il ne peut être porté atteinte à ce droit qu'en conformité avec les principes de la justice fondamentale» (Charte canadienne des droits et libertés, L.R.C. (1985), Ap. II, nº 44, art. 7). La jurisprudence canadienne est aujourd'hui à l'effet que cette garantie du droit à la vie n'est opposable qu'aux actes de nature gouvernementale et ne s'applique pas dans le contexte des rapports de droit privé (Syndicat des détaillants, grossistes et magasins à rayons section locale 580 c. Dolphin Delivery Ltd., [1986] 2 R.C.S. 573). Il va de soi dans le cadre de la thèse défendue ici que cette restriction héritée du constitutionnalisme classique ne saurait être maintenue. Heureusement, d'autres instruments constitutionnels protégeant les droits fondamentaux ont un champ d'application qui couvre aussi bien les rapports privés que les relations entre l'État et les individus. C'est le cas pour la Charte québécoise des droits et libertés de la personne, L.R.Q., c. C-12, et notamment pour son article 1: «Tout être humain a droit à la vie, ainsi qu'à la sûreté, à l'intégrité et à la liberté de sa personne. " Pour une analyse des effets au moins indirects de la constitutionnalisation des droits de la personne humaine sur le droit des rapports privés, voir F. Allard, «L'impact de la Charte canadienne des droits et libertés sur le droit civil : une relecture de l'arrêt Dolphin Delivery à l'aide d'une réflexion sur les sources du droit civil québécois ", Revue du Barreau du Québec, numéro spécial, 2003, p. 1-72 (44sq.).

52. Sur le bénéfice que les personnes morales de droit privé ont tiré de la plupart des droits et libertés constitutionnellement garantis par les chartes des droits, voir T. Hartman, Unequal Protection. The Rise of Corporate Dominance and the Theft of Human Rights, Emmaüs, Rodale Press, 2002; C. J. Mayer, "Personalizing the impersonal : corporation and the bill of rights ", Hastings Law Journal, vol. 41, n 3, mars 1990, p. 664; J.-P. Gervais, « Les personnes morales et la Charte canadienne des droits et libertés», Revue de droit de McGill, 38, 1993, p. 263. Pour une critique de ce phénomène, voir M. Dan-Cohen, Rights, Persons, and Organizations. A Legal Theory of Bureaucratic Society, Berkeley, University of California Press, 1986. Cet accaparement des droits fondamentaux par les personnes morales s'est trouvé accéléré par la propagation d'un paradigme néolibéral des droits de la personne qui s'impose de plus en plus au paradigme humaniste de la Déclaration universelle des Droits de l'homme. Voir, à ce sujet, O. Onazi, «Towards a subaltern theory of Human Rights », Global Jurist Advances, vol. 9, $\mathrm{n}^{\circ}$ 2, 2009, p. 11 : « [...] this TRMF [trade-related market-friendly Human Rights regime] paradigm prioritizes the collective (human) rights of global capital over those of "flesh and blood" human beings. » L'auteur emprunte cette thèse à U. Baxi, The Future of Human Rights, Delhi - New York, Oxford University Press, 2002.

53. Entendu au sens étroit de la vie biologique, il va de soi que le droit à la vie ne peut être étendu aux organisations et personnes morales puisqu'elles n'ont qu'un corps métaphorique. La discrimination prônée ici prend toute sa valeur dès lors que le droit à la vie est entendu dans une acception qui déborde largement ce sens étroit comme nous le proposons. On peut évidemment imaginer une discrimination moins radicale qui laisserait place à la reconnaissance d'une personnalité juridique (et d'un droit à la vie limité?) aux attributions moins étendues s'agissant des personnes morales. En contrepartie, ces dernières se verraient imposer, comme l'État moderne, la responsabilité de garantir et promouvoir le respect des droits fondamentaux des êtres humains. Voir, en ce sens, D. Kinley et D. Tadaki, «From talk to walk: the emergence of Human Rights responsibilities for corporations at international law », Virginia Journal of International Law, vol. 44, $\mathrm{n}^{\circ}$ 4, 2003/4, p. 945-946. 
social; le droit à la subsistance, à une vie matérielle décente, même si cela exige de la collectivité la mise en œuvre d'une justice distributive qui régresse aujourd'hui au sein des nations les plus riches et qui attend encore son heure à l'échelle planétaire; le droit à la vie écologique, à un environnement durable, à défaut de quoi l'existence humaine ne se maintient qu'en sursis, comme une espèce en voie de disparition parmi d'autres; le droit à la vie de l'esprit et à la diversité culturelle pour qu'il reste des âmes humaines concrètes à opposer à l'homogénéisation matérialiste et techniciste accélérée par la mondialisation; le droit à la vie démocratique, enfin, pour que les individus restent en principe et en pratique au centre des décisions stratégiques, à la faveur de dispositions protectrices qui excluront fermement l'expression politique des personnes morales et les confineront à un strict rôle opérationnel ${ }^{54}$.

Ce n'est pas demain la veille, admettons-le, que ces dimensions déjà affirmées dans la culture juridique moderne jouiront d'une pleine protection de principe dans le droit en vigueur et d'une effectivité concrète dans l'action et la régulation des organisations et systèmes. Paradoxalement, c'est peut-être en exigeant encore davantage, au nom d'un droit à la vie étendu au-delà de son acception juridique actuelle, que la conscience sociale humaniste réussira le mieux à soutenir les avancées juridiques de la modernité en faveur de la prééminence du principe humain sur le principe fonctionnel.

Pensons dans l'utopie deux dimensions juridiquement nouvelles du droit à la vie qu'il s'agira de faire pré- valoir sur le droit de l'utilité, de l'efficacité et de l'efficience organisationnelles. Premièrement, le droit à la vie affective, le droit protégeant l'attachement humain à autrui, à la nature, au territoire, à la tradition, le droit de préférer l'enracinement à la mobilité, le statu quo au progrès, la décroissance à la croissance, le droit à la vie simple plutôt qu'augmentée. Deuxièmement, le droit à la vie inutile, le droit au détachement humain, le droit à l'évasion et à la solitude, le droit à la dissolution volontaire du moi personnel, le droit de casser le miroir qui ne renvoie à l'être humain que l'image de ses masques à usage social, et ultimement, parce qu'il est inclus fatalement dans le droit à la vie, le droit de mourir volontairement à soi et conséquemment aux autres, le droit de risquer le saut définitif dans un nuage où l'esprit humain s'abandonne à un cosmos plus grand que lui.

Garantir juridiquement et socialement, avec droit de retrait et compensation financière, la liberté de préférer l'affection à la fonction, l'indisponibilité à l'utilité, telle serait la condition à satisfaire ultimement pour que toutes les autres dimensions du droit à la vie prennent leur sens plénier et soient confirmées sans compromis dans leur autorité normative. Ce rêve utopique mais nécessaire participe lui-même du droit fondamental à la vie. Sans ce devoir-être premier, il est illusoire d'espérer que le respect de la dignité humaine s'impose à la promotion du pluralisme juridique ${ }^{55}$.

54. Le droit à la participation démocratique et le contrôle des êtres humains sur les décisions collectives ne sauraient logiquement et pratiquement se restreindre aux seules organisations étatiques. La jonction du pluralisme juridique et de la promotion des droits de la personne suppose que les êtres humains bénéficient d'une démocratie animée par eux aussi bien dans les organisations privées, y compris à but lucratif, que dans les autres, au niveau mondial aussi bien que local. Dans un pluralisme juridique favorable aux droits des êtres humains, la participation démocratique préside à la création et à la gestion du droit propre à toute organisation. Voir, en ce sens, O. Onazi, «Towards a subaltern theory of Human Rights », p. 16-22.

55. Je dédie ce texte à la mémoire de mon père. Loyal agent d'une grande société d'assurances, il fut néanmoins un homme de cœur bien davantage qu'un «homme de l'organisation». 
